\title{
Explicit Control Force and Torque Determination for Satellite Formation-Keeping with Attitude Requirements
}

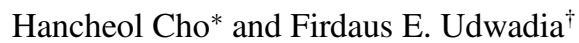 \\ University of Southern California, Los Angeles, California 90089
}

DOI: $10.2514 / 1.55873$

\begin{abstract}
A simple analytical approach for formation-keeping of satellites in the presence of both orbital and attitude requirements is developed. A leader satellite is assumed to be in a $\boldsymbol{J}_{\mathbf{2}}$-perturbed circular reference orbit and each follower satellite is required to stay in its prescribed, desired orbit with respect to the leader satellite, and at the same time, to point to an arbitrarily chosen specific spot in space that may be time-varying. Nonlinear relative dynamics is considered in its entirety without any approximations, and nonlinear controllers are designed to exactly satisfy the desired orbital and attitude mission requirements for the formation. The analytical approach leads to a separation principle that points out when orbital control is completely unaffected by attitude control. In the presence of sensor measurement noise that leads to incorrect initial conditions at the start of the control maneuver the approach asymptotically satisfies both the orbital and attitude control requirements, at any desired rate of convergence depending on mission requirements. The effect of small unmodelled dynamics is also considered. A numerical example demonstrates the simplicity of the approach, its ease of implementation, accuracy of results, and its robustness to both sensor measurement errors and unmodelled dynamics.
\end{abstract}

\section{Introduction}

$\mathbf{T}$ HE use of formation flight is expected to afford considerable advantages for certain space missions when compared with existing space technology. The configured flight of small multiple satellites holds out the potential for reducing total mission costs, performing certain missions more flexibly and efficiently, and making possible many advanced applications such as space interferometry and high-resolution imaging [1] . However, formation flying requires technology from various research fields, such as relative orbit determination, formation-keeping, formation reconfiguration, relative attitude determination, and relative attitude control. Among these, in this paper, the formation-keeping problem is considered. Briefly speaking, formation-keeping is the technique to accurately maintain a desired formation geometry. The aim is to control each follower satellite (the satellite around the leader satellite) to achieve a useful formation configuration with the capability of precision attitude control. The leader satellite may be a satellite moving along a predefined orbit, or it may be a fictitious satellite serving as a reference with respect to which a "swarm" of satellites is required to fly in formation. The leader satellite's orbit shall be referred to as the reference orbit. The relative trajectories of the follower satellites (both with regard to their orbital requirements and their attitude requirements) that comprise the formation may be static in time, or they may be required to change dynamically in some arbitrarily prescribed manner.

Because formation-keeping is important to successfully achieve a mission goal by regulating the orbits and attitudes of the satellites that fly in a cluster, numerous researchers have been attracted to this problem. Most of the concern to date, however, has been focused only on orbital dynamics, much less on attitude dynamics. Traditionally, linear control theories have been used, based on linearized equations of relative motion, such as a Hill-Clohessy-Wiltshire (HCW) equation $[2, \underline{3}]$ for a circular reference orbit, a Tschauner-Hempel

Received 5 August 2011; revision received 3 February 2012; accepted for publication 15 April 2012; published online 1 January 2013. Copyright (C) 2012 by the American Institute of Aeronautics and Astronautics, Inc. All rights reserved. Copies of this paper may be made for personal or internal use, on condition that the copier pay the $\$ 10.00$ per-copy fee to the Copyright Clearance Center, Inc., 222 Rosewood Drive, Danvers, MA 01923; include the code 1533-3884/13 and \$10.00 in correspondence with the CCC.

*Graduate Student, Department of Aerospace and Mechanical Engineering.

${ }^{\dagger}$ Professor, Departments of Aerospace and Mechanical Engineering, Civil Engineering, Mathematics, Systems Architecture Engineering, and Information and Operations Management, 430K Olin Hall. equation [4] for an elliptical reference orbit, or the $J_{2}$-perturbed linearized equation [5]. Yan et al. [6] designed a linear quadratic regulator (LQR) for the satellites' periodic motion based on the HCW equation. Sparks [7] studied the same problem and considered nonspherical gravity perturbations. Using the HCW equation, Vaddi and Vadali [8] presented Lyapunov, LQR, and period-matching control schemes that generate a projected circular orbit (PCO), which is also chosen as the desired formation geometry in this paper. They showed that the LQR method offers cost benefits, but guarantees only local stability compared with the others. Won and Ahn [9] assumed an elliptical reference orbit to develop the state-dependent Riccati equation control technique for formation-keeping with constant separation distance between satellites. Because the solutions based on the linearized equations about Keplerian orbits are suitable only for very small-sized formations due to the linearizations used, Milam et al. [10] used a numerical solver, called the nonlinear trajectory generation software package, to find an optimal controller for formation-keeping. Based on nonlinear dynamics describing the relative motion around a circular reference orbit, De Queiroz et al. [11] developed an adaptive controller for tracking the relative position. They showed that global asymptotic position tracking errors are guaranteed even in the presence of unknown slow-varying perturbation forces. No et al. [12] started with general nonlinear equations of motion to develop closed-form equations using power series and trigonometric functions, which enable a series of optimal impulsive maneuvers to maintain a formation configuration.

A few researchers have incorporated attitude dynamics in formation flying. Kristiansen et al. [13] derived a nonlinear model describing relative translational and rotational motion. In [14], a controller that uses Lyapunov control theory was introduced for target tracking while countering the effect of gravity gradient torques. Lennox [15] first separately designed two nonlinear Lyapunov controllers for orbital and attitude control and then integrated them into a coupled control strategy. Unlike others, he assumed that orbital control is dependent on the satellite's attitude for vectored thrust. Ahn and $\mathrm{Kim}$ [16] assumed a formation of satellites as a virtual rigid-body structure to develop an algorithm for pointing to a specified target. They combined an adaptive control scheme and a sliding mode control scheme to make the satellites follow the desired position and attitude command.

Previous work is thus seen to mainly focus on linearized approaches and, when nonlinear dynamics is considered, the controllers for formation-keeping have been obtained via the Lyapunov method or by numerical approaches. Recently, a nonlinear controller that preserves the nonlinearities of the governing dynamics 
was proposed by Udwadia and Kalaba [17-26]. It has a surprisingly simple, explicit form and is applicable to a number of cases that have holonomic and/or nonholonomic constraints. Also, no a priori structure is imposed on this nonlinear controller, as is commonly the case. Because of its simplicity and generality, this method has been applied to highly constrained problems, such as control of tethered satellites [27] or orbit transfers under perturbations [28]. For the formation-keeping problem, using this approach, Cho and Yu [29] obtained an analytical solution for formation-keeping when the leader satellite is in an unperturbed circular orbit. The follower satellites were constrained to lie in a PCO, and the problem was solved in the inertial frame first, then transformed into the Hill frame for better visualization. Cho and Udwadia [30] extended this solution to more general cases in which the leader satellite follows any arbitrary orbit and the follower satellites are not initially on the desired trajectory, thereby taking into account errors that arise during orbit insertion. The problem was solved in the Hill frame, not in the inertial frame, because measurements in formation flying are often taken in the Hill frame or in the so-called local vertical, local horizontal (LVLH) frame. References $[29,30]$ did not, however, consider any attitude dynamics. Udwadia et al. [31] solved the precision tumbling and precision tracking problems for a nonlinear, nonautonomous, multibody system orbiting a central body with a nonuniform gravity field. They included attitude dynamics and used a closed-form control methodology to achieve "exact" control. The inertial frame was used and the vibrating nonlinear multibody system was required to be in a precise circular orbit around the central body, while it underwent arbitrarily prescribed on-orbit precision tumbling.

This paper extends the results of [29-31] to develop a closed-form nonlinear controller when the leader satellite follows a $J_{2}$-perturbed circular reference orbit and the follower satellites point to a specific spot in space during the maneuver. Each follower may be required to track a different spot, which may be arbitrarily specified and could be time varying. The satellites are considered as rigid bodies and, for describing the attitude dynamics quaternions, are used so that arbitrary orientations can be realized while avoiding singularities. Using suitable generalized coordinates, it is shown that both orbital and attitude control can be easily handled in a simple and unified way, yielding closed-form results for the control forces and the control torques that can be used for real-time control. Numerical simulations are performed to show the simplicity and accuracy of the approach developed herein. One of the strengths of this paper lies in the fact that the closed-form solutions obtained to the nonlinear problem (both in terms of the dynamics and the control) permit some important physical insights into the nature of the control, something that would be difficult to obtain from purely computational approaches. For example, in the current paper, conditions under which orbital control is independent of the attitude of the satellite, so that one need not consider any attitude or quaternions in designing the orbital controller, are investigated. The methodology permits the inclusion of sensor measurement errors that result in inaccurate initial conditions at the start of the maneuver. This specific problem has been addressed by several investigators $[32,33]$ who showed the strong sensitivity of currently used control methods (only orbital control has been considered in these references) to such errors. In the presence of such measurement errors, the approach presented herein causes the orbital and attitude requirements to be satisfied asymptotically at a rate that can be adjusted, depending on the specific mission requirements. Finally, the effect of unmodeled dynamics is considered and it is shown that, by augmenting the closed-form nonlinear controller obtained herein with a simple proportional-derivative (PD) controller, the control requirements can be met with high accuracy, thereby pointing out the robustness with which unmodeled dynamics can be easily handled.

The paper is organized in the following manner. In Sec. II, the fundamental equations of motion for constrained systems are briefly introduced, and the explicit closed-form expressions for the control forces are followed. Section III deals with the unconstrained orbital and attitude motions separately. Equations of motion are derived for translational and rotational dynamics when no control is applied, and they are later combined to handle the control problem in a unified way and to obtain explicit expressions for the control forces and torques. The methodology is applicable to the situation in which there is sensor measurement noise that leads to incorrect initial conditions at the start of the control maneuver. In contrast to other control methods that show high sensitivity to such errors, the approach provided here is shown to asymptotically satisfy both the orbital and the attitude requirements. Because the control forces and torques are obtained in closed form, it is very easy and fast to implement these results in real time when making space maneuvers. Insights into the dynamics lead to the separation principle, which is described in Sec. IV. Finally, in Sec. V, a specific example is presented to demonstrate the results and show the efficacy and the accuracy of the closed-form method. The example shows that, even in the presence of sensor measurement noise, the exact control requirements are quickly met. It also demonstrates the ease of obtaining robust control in the presence of unmodeled dynamics by augmenting the nonlinear closed-form controller obtained herein with simple PD control.

\section{Fundamental Equations}

This section deals with the explicit equation of motion for a constrained mechanical system. First, let us consider an unconstrained, discrete dynamic system of $N$ rigid bodies (or satellites in this paper) in generalized coordinates. The generalized displacement vector and velocity vector are denoted by $\mathbf{q}(t)$ and $\dot{\mathbf{q}}(t)$, respectively,

$$
\begin{aligned}
\mathbf{q}(t) & =\left[\begin{array}{llll}
q_{1}(t) & q_{2}(t) & \cdots & q_{n}(t)
\end{array}\right]^{\mathbf{T}}, \\
\dot{\mathbf{q}}(t) & =\left[\begin{array}{llll}
\dot{q}_{1}(t) & \dot{q}_{2}(t) & \cdots & \dot{q}_{n}(t)
\end{array}\right]^{\mathbf{T}}
\end{aligned}
$$

where $t$ represents time, the superscript $\mathbf{T}$ denotes the transpose of a vector or a matrix, and $n$ is the number of the generalized coordinates. If the given forces impressed on the bodies are denoted by

$$
\mathbf{Q}(t)=\left[\begin{array}{llll}
Q_{1}(t) & Q_{2}(t) & \cdots & Q_{n}(t)
\end{array}\right]^{\mathbf{T}}
$$

then the Lagrange equation for the unconstrained motion of the system at a certain instant of time $t$ can now be expressed as an $n \times 1$ matrix equation

$$
\mathbf{M} \ddot{\mathbf{q}}(t)=\mathbf{Q}[\mathbf{q}(t), \dot{\mathbf{q}}(t), t]
$$

or

$$
\ddot{\mathbf{q}}(t)=\mathbf{M}^{-1} \mathbf{Q}[\mathbf{q}(t), \dot{\mathbf{q}}(t), t]:=\mathbf{a}(t)
$$

where $\mathbf{M}$ (which is positive definite in this paper) is an $n \times n$ symmetric mass matrix and $\mathbf{a}(t)$ is the $n \times 1$ unconstrained acceleration (vector) of the system at time $t$.

It is now considered that the unconstrained system described by Eqs. (3) and (4) is subjected to $p$ constraints, which are of the form

$$
\varphi_{j}(\mathbf{q}, \dot{\mathbf{q}}, t)=0, \quad j=1,2, \cdots, p
$$

These $p$ constraints include both orbital and attitude requirements for precise formation-keeping in the current paper. More compactly, one can write Eq. (5) as follows

$$
\boldsymbol{\Phi}=\left[\begin{array}{llll}
\varphi_{1} & \varphi_{2} & \cdots & \varphi_{p}
\end{array}\right]^{\mathbf{T}}=0
$$

Differentiating Eq. (6) with respect to time once (for nonholonomic constraints) or twice (for holonomic constraints) yields the following constraint equation

$$
\mathbf{A}[\mathbf{q}(t), \dot{\mathbf{q}}(t), t] \ddot{\mathbf{q}}(t)=\mathbf{b}[\mathbf{q}(t), \dot{\mathbf{q}}(t), t]
$$

where the matrix $\mathbf{A}$ is a $p \times n$ matrix and $\mathbf{b}$ is a $p \times 1$ vector. However, because the initial conditions must be determined from measurements, and they will be corrupted by sensor noise or unmodeled dynamics, it is in general difficult to make them satisfy the given orbital/attitude constraints at the initial time, which means $\boldsymbol{\Phi}(t=0) \neq 0$, and so the constraint equations (6) should be modified to $[\underline{23}, \underline{34}]$ 


$$
\ddot{\boldsymbol{\Phi}}+\boldsymbol{\alpha} \dot{\boldsymbol{\Phi}}+\boldsymbol{\beta} \boldsymbol{\Phi}=0
$$

where

$$
\boldsymbol{\alpha}=\operatorname{diag}\left\{\alpha_{1}, \alpha_{2}, \cdots, \alpha_{p}\right\}, \quad \boldsymbol{\beta}=\operatorname{diag}\left\{\beta_{1}, \beta_{2}, \cdots, \beta_{p}\right\}
$$

By choosing each $\alpha_{j}, \beta_{j}>0, j=1,2, \cdots, p$, $\boldsymbol{\Phi}$ (i.e., each constraint) approaches zero asymptotically. Thus, from Eq. ()ㅡ, one can get a more general constraint equation, which nonetheless retains the form of Eq. (7).

The presence of the constraints (6) causes additional forces $\mathbf{Q}^{\mathbf{C}}(t)$ to be applied to the bodies, and the resulting equation of motion becomes [17]

$$
\mathbf{M} \ddot{\mathbf{q}}(t)=\mathbf{Q}[\mathbf{q}(t), \dot{\mathbf{q}}(t), t]+\mathbf{Q}^{\mathbf{C}}(t)
$$

where the (constraint) force $\mathbf{Q}^{\mathbf{C}}(t)$ makes the system asymptotically satisfy the given constraints of Eq. (6). Historically, there have been a number of attempts to determine $\vec{Q}^{\mathbf{C}}(t)$ and, recently, a simple and insightful approach was proposed by Udwadia and Kalaba [17-26], which gives the constraint force $\mathbf{Q}^{\mathbf{C}}(t)$ explicitly as

$$
\mathbf{Q}^{\mathbf{C}}(t)=\mathbf{M}^{1 / 2}\left(\mathbf{A M}^{-1 / 2}\right)^{+}(\mathbf{b}-\mathbf{A} \mathbf{a})
$$

or, equivalently,

$$
\mathbf{Q}^{\mathbf{C}}(t)=\mathbf{A}^{\mathbf{T}}\left(\mathbf{A} \mathbf{M}^{-1} \mathbf{A}^{\mathbf{T}}\right)^{+}(\mathbf{b}-\mathbf{A} \mathbf{a})
$$

where the superscript + represents the Moore-Penrose (MP) generalized inverse.

Thus, the explicit equation of motion of the constrained system is the following

$$
\mathbf{M} \ddot{\mathbf{q}}=\mathbf{M a}+\mathbf{M}^{1 / 2}\left(\mathbf{A} \mathbf{M}^{-1 / 2}\right)^{+}(\mathbf{b}-\mathbf{A} \mathbf{a})
$$

or

$$
\mathbf{M} \ddot{\mathbf{q}}=\mathbf{M a}+\mathbf{A}^{\mathbf{T}}\left(\mathbf{A} \mathbf{M}^{-1} \mathbf{A}^{\mathbf{T}}\right)^{+}(\mathbf{b}-\mathbf{A a})
$$

Accordingly, the acceleration of the system is obtained by

$$
\ddot{\mathbf{q}}=\mathbf{a}+\mathbf{M}^{-1 / 2}\left(\mathbf{A M}^{-1 / 2}\right)^{+}(\mathbf{b}-\mathbf{A} \mathbf{a})
$$

or

$$
\ddot{\mathbf{q}}=\mathbf{a}+\mathbf{M}^{-1} \mathbf{A}^{\mathbf{T}}\left(\mathbf{A} \mathbf{M}^{-1} \mathbf{A}^{\mathbf{T}}\right)^{+}(\mathbf{b}-\mathbf{A a})
$$

In this paper, Eq. (13) are referred to as the fundamental equations, and the constraint force $\mathbf{Q}^{\mathbf{C}}(t)$ in Eq. (11) is used for controlling the orbital and/or attitude motion of the follower satellites, so that each of them satisfies its formation-keeping requirements. Furthermore, it is shown in [23] that this constraint force $\mathbf{Q}^{\mathbf{C}}(t)$ minimizes at each instant of time $t$ the quantity $\left[\mathbf{Q}^{\mathbf{C}}(t)\right]^{\mathbf{T}} \mathbf{M}^{-1}(\mathbf{q}, t)\left[\mathbf{Q}^{\mathbf{C}}(t)\right]$. In what follows, the arguments of the various quantities are usually suppressed, as in Eqs. (12) and (13), unless needed for clarity. Also, the term "unconstrained motion" shall be used to mean "uncontrolled motion," and the term "constrained motion" shall mean "controlled motion."

\section{Formation-Keeping Equations of Motion}

It is assumed that there are $N$ follower satellites and that a leader satellite leads this $N$-satellite formation. The $i$ th follower satellite has a mass $m^{(i)}$ and has a diagonal inertia matrix $\mathbf{J}^{(i)}$, whose entries give the moments of inertia along the body-fixed principal axes of inertia, whose origin is located at the center of mass of the $i$ th follower satellite. In what follows, the superscript $i$ shall be used to denote quantities relevant to the $i$ th follower satellite.

In this paper, three different coordinate frames of reference are used: an inertial frame, a Hill frame, and a body-fixed frame. The inertial frame, more precisely the ECI (Earth-centered inertial) frame, originates at the center of the Earth, the $X$ axis points toward the vernal equinox, the $Y$ axis is $90 \mathrm{deg}$ to the east in the equatorial plane, and the $Z$ axis extends through the North Pole [35]. Next, the Hill frame is useful when describing the relative motion of satellites in formation. The origin of this frame is located at the leader satellite that moves in a specified reference orbit, the $x$ axis is directed radially outward along the local vertical, the $z$ axis is along the instantaneous orbital angular momentum vector of the leader satellite, and the $y$ axis is taken perpendicular to the $x z$ plane, to form a right-handed triad. Lastly, a different body-fixed frame is used to describe the attitude motion of each follower satellite. Each axis of the body-fixed frame of the $i$ th follower satellite points along its principal axes of inertia, and the origin of this frame is located at its center of mass.

It is assumed that the position vector of the center of mass of the $i$ th follower satellite in the ECI frame is given by the three vector $\mathbf{X}^{(i)}=\left[\begin{array}{lll}X^{(i)} & Y^{(i)} & Z^{(i)}\end{array}\right]^{\mathbf{T}}$, and its orientation is described by the quaternion four vector $\mathbf{u}^{(i)}=\left[\begin{array}{llll}u_{0}^{(i)} & u_{1}^{(i)} & u_{2}^{(i)} & u_{3}^{(i)}\end{array}\right]^{\mathbf{T}}$. The generalized displacement seven vector is then defined as

$$
\begin{gathered}
\mathbf{q}^{(i)}(t):=\left[\begin{array}{lllllll}
X^{(i)} & Y^{(i)} & Z^{(i)} & u_{0}^{(i)} & u_{1}^{(i)} & u_{2}^{(i)} & u_{3}^{(i)}
\end{array}\right]^{\mathbf{T}}, \\
\quad i=1,2, \cdots, N
\end{gathered}
$$

\section{A. Uncontrolled Orbital Motion}

The inertial orbital motion of the $i$ th follower satellite, perturbed by the second zonal harmonics, is governed by the relation [36]

$$
\begin{aligned}
\mathbf{a}^{(i)} & =\ddot{\mathbf{X}}^{(i)}=\left[\begin{array}{c}
\ddot{X}^{(i)} \\
\ddot{Y}^{(i)} \\
\ddot{Z}^{(i)}
\end{array}\right]=-\frac{G M \bigoplus}{r^{(i)^{3}}}\left[\begin{array}{c}
X^{(i)} \\
Y^{(i)} \\
Z^{(i)}
\end{array}\right] \\
- & \frac{3}{2} J_{2}\left(\frac{G M \bigoplus}{r^{(i)^{2}}}\right)\left(\frac{R}{r^{(i)}}\right)^{2}\left[\begin{array}{l}
\left(1-5\left(\frac{Z^{(i)}}{r^{(i)}}\right)^{2}\right) \frac{X^{(i)}}{r^{(i)}} \\
\left(1-5\left(\frac{Z^{(i)}}{r^{(i)}}\right)^{2}\right) \frac{Y^{(i)}}{r^{(i)}} \\
\left(3-5\left(\frac{Z^{(i)}}{r^{(i)}}\right)^{2}\right) \frac{Z^{(i)}}{r^{(i)}}
\end{array}\right] \\
= & \mathbf{a}_{2-\text { body }}^{(i)}+\mathbf{a}_{J_{2}}^{(i)}
\end{aligned}
$$

where $\mathbf{X}^{(i)}=\left[\begin{array}{lll}X^{(i)} & Y^{(i)} & Z^{(i)}\end{array}\right]^{\mathbf{T}}$ is the position vector of the center of mass of the $i$ th follower satellite in the ECI frame, $r^{(i)}=$ $\sqrt{X^{(i)^{2}}+Y^{(i)^{2}}+Z^{(i)^{2}}}$ is the distance from the center of the Earth to the center of mass of the $i$ th follower satellite, $G$ is the universal gravitational constant, $M_{\bigoplus}$ is the mass of the Earth, $R_{\bigoplus}$ is the mean equatorial radius of the Earth, and $J_{2}=1.0826269 \times 10^{-3}$ is the coefficient of the second zonal harmonics of the Earth's potential. In the right-hand side of Eq. (15), the first term $\left(\mathbf{a}_{2-\text { body }}^{(i)}\right)$ describes twobody motion and the second term $\left(\mathbf{a}_{J_{2}}^{(i)}\right)$ corresponds to the perturbing acceleration due to $J_{2}$.

Next, let us recall the transformation between the ECI frame and the Hill frame [30]:

$$
\left[\begin{array}{c}
x^{(i)}+r_{L} \\
y^{(i)} \\
z^{(i)}
\end{array}\right]=\mathbf{R}\left[\begin{array}{c}
X^{(i)} \\
Y^{(i)} \\
Z^{(i)}
\end{array}\right]:=\left[\begin{array}{l}
\mathbf{R}_{1} \\
\mathbf{R}_{2} \\
\mathbf{R}_{3}
\end{array}\right] X^{(i)}
$$

where $\mathbf{x}^{(i)}=\left[\begin{array}{lll}x^{(i)} & y^{(i)} & z^{(i)}\end{array}\right]^{\mathbf{T}}$ is the position vector of the center of mass of the $i$ th follower satellite in the Hill frame, $r_{L}$ is the distance from the center of the Earth to the leader satellite (the subscript $L$ denotes the leader satellite), and $\mathbf{R}$ is a $3 \times 3$ orthogonal rotation matrix, whose row vectors are $\mathbf{R}_{1}, \mathbf{R}_{2}$, and $\mathbf{R}_{3}$. The matrix $\mathbf{R}$ includes the information about the leader satellite's orbit and each component of the matrix can be found in [30]. 


\section{B. Uncontrolled Rotational Motion}

As mentioned, rotational dynamics of each follower satellite is described in terms of quaternions that expel the troublesome problem of singularity. Here, the method given in [37] is adopted.

Let us begin with the Lagrange equation

$$
\frac{\mathrm{d}}{\mathrm{d} t}\left(\frac{\partial T^{(i)}}{\partial \dot{\mathbf{u}}^{(i)}}\right)-\frac{\partial T^{(i)}}{\partial \mathbf{u}^{(i)}}=\Gamma_{\mathbf{u}}^{(i)}\left(\mathbf{u}^{(i)}, \dot{\mathbf{u}}^{(i)}, t\right)
$$

where $\mathbf{u}^{(i)}=\left[\begin{array}{llll}u_{0}^{(i)} & u_{1}^{(i)} & u_{2}^{(i)} & u_{3}^{(i)}\end{array}\right]^{\mathbf{T}}$ is the quaternion four vector of the $i$ th follower satellite, $\boldsymbol{\Gamma}_{\mathbf{u}}^{(i)}\left(\mathbf{u}^{(i)}, \dot{\mathbf{u}}^{(i)}, t\right)$ is the given generalized force vector, and $T^{(i)}$ is the rotational kinetic energy of the $i$ th follower satellite, which is defined by

$$
T^{(i)}:=\frac{1}{2}\left\{\omega^{(i)}\right\}^{\mathrm{T}} \hat{\mathbf{J}}^{(i)}\left\{\boldsymbol{\omega}^{(i)}\right\}
$$

where the four vector $\left\{\boldsymbol{\omega}^{(i)}\right\}=\left[\begin{array}{llll}0 & \omega_{x}^{(i)} & \omega_{y}^{(i)} & \omega_{z}^{(i)}\end{array}\right]^{\mathbf{T}}=$ $\left[\begin{array}{ll}0 & \boldsymbol{\omega}^{(i)}\end{array}\right]^{\mathbf{T}}$ is defined and the three vector $\boldsymbol{\omega}^{(i)}=$ $\left[\begin{array}{lll}\omega_{x}^{(i)} & \omega_{y}^{(i)} & \omega_{z}^{(i)}\end{array}\right]^{\mathbf{T}}$ comprises the components of the angular velocity of the $i$ th follower satellite along its body-fixed coordinate axes. Also, the $4 \times 4$ augmented inertia matrix, $\hat{\mathbf{J}}^{(i)}>0$, is defined as [37]

$$
\hat{\mathbf{J}}^{(i)}:=\left[\begin{array}{cc}
J_{0}^{(i)} & 0 \\
0 & \mathbf{J}^{(i)}
\end{array}\right]=\left[\begin{array}{cccc}
J_{0}^{(i)} & 0 & 0 & 0 \\
0 & J_{x}^{(i)} & 0 & 0 \\
0 & 0 & J_{y}^{(i)} & 0 \\
0 & 0 & 0 & J_{z}^{(i)}
\end{array}\right]
$$

where $J_{0}^{(i)}$ is an arbitrary positive number, and $J_{x}^{(i)}, J_{y}^{(i)}$, and $J_{z}^{(i)}$ are the moments of inertia along the principal axes of the $i$ th follower satellite. The augmented angular velocity four vector $\left\{\boldsymbol{\omega}^{(i)}\right\}$, mentioned in Eq. (18), is related with quaternions by

$$
\left\{\boldsymbol{\omega}^{(i)}\right\}=2 \mathbf{E}^{(i)} \dot{\mathbf{u}}^{(i)}, \quad i=1,2, \cdots, N
$$

The $4 \times 4$ quaternion matrix $\mathbf{E}^{(i)}$ that is orthogonal is defined by

$$
\mathbf{E}^{(i)}:=\left[\begin{array}{c}
\mathbf{u}^{(i)^{\mathbf{T}}} \\
\mathbf{E}_{1}^{(i)}
\end{array}\right]=\left[\begin{array}{cccc}
u_{0}^{(i)} & u_{1}^{(i)} & u_{2}^{(i)} & u_{3}^{(i)} \\
-u_{1}^{(i)} & u_{0}^{(i)} & u_{3}^{(i)} & -u_{2}^{(i)} \\
-u_{2}^{(i)} & -u_{3}^{(i)} & u_{0}^{(i)} & u_{1}^{(i)} \\
-u_{3}^{(i)} & u_{2}^{(i)} & -u_{1}^{(i)} & u_{0}^{(i)}
\end{array}\right]
$$

Substituting Eq. (20) into Eq. (18) yields the rotational kinetic energy in terms of quaternions

$$
T^{(i)}=2 \dot{\mathbf{u}}^{(i)^{\mathbf{T}}} \mathbf{E}^{(i)^{\mathbf{T}}} \hat{\mathbf{J}}^{(i)} \mathbf{E}^{(i)} \dot{\mathbf{u}}^{(i)}
$$

Then, assuming that there is no applied torque (i.e., $\boldsymbol{\Gamma}_{\mathbf{u}}^{(i)}=0$ ), one can apply the Lagrange equation under the assumption that the components of the quaternion four vector are all independent of each other, to obtain

$$
4 \mathbf{E}^{(i)^{\mathbf{T}}} \hat{\mathbf{J}}^{(i)} \mathbf{E}^{(i)} \ddot{\mathbf{u}}^{(i)}=-8 \dot{\mathbf{E}}^{(i)^{\mathbf{T}}} \hat{\mathbf{J}}^{(i)} \mathbf{E}^{(i)} \dot{\mathbf{u}}^{(i)}-4 \mathbf{E}^{(i)^{\mathbf{T}}} \hat{\mathbf{J}}^{(i)} \dot{\mathbf{E}}^{(i)} \dot{\mathbf{u}}^{(i)}
$$

This relation can be written in the form of $\mathbf{M}_{\mathbf{u}}^{(i)} \ddot{\mathbf{u}}^{(i)}=\mathbf{Q}_{\mathbf{u}}^{(i)}$ by setting $\mathbf{M}_{\mathbf{u}}^{(i)}=4 \mathbf{E}^{(i)^{\mathrm{T}}} \hat{\mathbf{J}}^{(i)} \mathbf{E}^{(i)}$, and $\mathbf{Q}_{\mathbf{u}}^{(i)}$ to be the right-hand side of Eq. (23). It is noted that the mass matrix $\mathbf{M}_{\mathbf{u}}^{(i)}=4 \mathbf{E}^{(i)^{\mathrm{T}}} \hat{\mathbf{J}}^{(i)} \mathbf{E}^{(i)}$ is symmetric and positive definite, and so it always has its inverse.

It is important to stress that, up until now, it has been assumed that each component of the quaternion vector $\mathbf{u}^{(i)}$ is independent of the others. However, to represent a physical rotation of a rigid body, the quaternion vector $\mathbf{u}^{(i)}$ must have unit norm, so that

$$
\left\|\mathbf{u}^{(i)}\right\|^{2}=u_{0}^{(i)^{2}}+u_{1}^{(i)^{2}}+u_{2}^{(i)^{2}}+u_{3}^{(i)^{2}}=1
$$

After by differentiating twice, the following constraint equation of the form of Eq. (7) is obtained:

$$
2\left[\begin{array}{llll}
u_{0}^{(i)} & u_{1}^{(i)} & u_{2}^{(i)} & u_{3}^{(i)}
\end{array}\right]\left[\begin{array}{c}
\ddot{u}_{0}^{(i)} \\
\ddot{u}_{1}^{(i)} \\
\ddot{u}_{2}^{(i)} \\
\ddot{u}_{3}^{(i)}
\end{array}\right]=-2\left(\dot{u}_{0}^{(i)^{2}}+\dot{u}_{1}^{(i)^{2}}+\dot{u}_{2}^{(i)^{2}}+\dot{u}_{3}^{(i)^{2}}\right)
$$

so that

$$
\begin{aligned}
& \mathbf{A}_{\mathbf{u}}^{(i)}=2\left[\begin{array}{llll}
u_{0}^{(i)} & u_{1}^{(i)} & u_{2}^{(i)} & u_{3}^{(i)}
\end{array}\right], \\
& b_{u}^{(i)}=-2\left(\dot{u}_{0}^{(i)^{2}}+\dot{u}_{1}^{(i)^{2}}+\dot{u}_{2}^{(i)^{2}}+\dot{u}_{3}^{(i)^{2}}\right):=-2 N\left(\dot{\mathbf{u}}^{(i)}\right)
\end{aligned}
$$

The resulting rotational equation of motion for the $i$ th follower satellite is thus given by the fundamental equations (13)

$$
\ddot{\mathbf{u}}^{(i)}=-\frac{1}{2} \mathbf{E}_{1}^{(i)^{\mathbf{T}}} \mathbf{J}^{(i)^{-1}}\left[\tilde{\boldsymbol{\omega}}^{(i)}\right] \mathbf{J}^{(i)} \boldsymbol{\omega}^{(i)}-N\left(\dot{\mathbf{u}}^{(i)}\right) \mathbf{u}^{(i)}
$$

where $\mathbf{E}_{1}^{(i)}, \mathbf{J}^{(i)}$, and $N\left(\dot{\mathbf{u}}^{(i)}\right)$ are defined in Eqs. (19), (21), and (26), respectively, and $\left[\tilde{\boldsymbol{\omega}}^{(i)}\right]$ is a skew-symmetric matrix defined by

$$
\left[\tilde{\boldsymbol{\omega}}^{(i)}\right]:=\left[\begin{array}{ccc}
0 & -\omega_{z}^{(i)} & \omega_{y}^{(i)} \\
\omega_{z}^{(i)} & 0 & -\omega_{x}^{(i)} \\
-\omega_{y}^{(i)} & \omega_{x}^{(i)} & 0
\end{array}\right]
$$

In Eq. (27), the attitude equation of motion has been described by the eighth-order system of differential equations using the quaternion and its derivative eight vector $\left(\mathbf{u}^{(i)}, \dot{\mathbf{u}}^{(i)}\right)$ instead of a seventh-order system that consists of the quaternion four vector and the body-fixed angular velocity three vector $\left(\mathbf{u}^{(i)}, \boldsymbol{\omega}^{(i)}\right)$. It is straightforward to switch from one to the other using Eq. (20), but the Lagrangian formulation has been preferred here for its uniformity.

\section{Controlled Dynamics of Coupled Orbital and Rotational Motion}

In this subsection, the orbital and attitude dynamics are combined. Defining the generalized displacement seven vector $\mathbf{q}^{(i)}(t)$ as in Eq. (14), one can obtain the following equation of unconstrained motion for each follower satellite from Eqs. (15) and (27)

$$
\begin{aligned}
& \mathbf{a}^{(i)}(t):=\left[\begin{array}{c}
\mathbf{a}_{1}^{(i)} \\
\mathbf{a}_{2}^{(i)}
\end{array}\right]=\left(\mathbf{M}^{(i)}\right)^{-1} \mathbf{Q}^{(i)}
\end{aligned}
$$

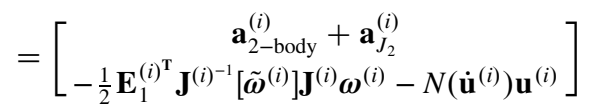

where $\mathbf{a}_{1}^{(i)}=\mathbf{a}_{2-\text { body }}^{(i)}+\mathbf{a}_{J_{2}}^{(i)}$ is a three vector on the right-hand side of Eq. (15), and $\mathbf{a}_{2}^{(i)}=-\frac{1}{2} \mathbf{E}_{1}^{(i)^{\mathrm{T}}} \mathbf{J}^{(i)^{-1}}\left[\tilde{\boldsymbol{\omega}}^{(i)}\right] \mathbf{J}^{(i)} \boldsymbol{\omega}^{(i)}-N\left(\dot{\mathbf{u}}^{(i)}\right) \mathbf{u}^{(i)}$ is a four vector on the right-hand side of Eq. (27). Also, the $7 \times 7$ positive definite mass matrix of the $i$ th follower satellite $\mathbf{M}^{(i)}$ is defined as

$$
\mathbf{M}^{(i)}:=\left[\begin{array}{cc}
m^{(i)} \mathbf{I}_{3 \times 3} & 0_{3 \times 4} \\
0_{4 \times 3} & \mathbf{M}_{\mathbf{u}}^{(i)}
\end{array}\right]
$$

where $m^{(i)}$ is the mass of the $i$ th follower satellite, $\mathbf{I}_{3 \times 3}$ is the $3 \times 3$ identity matrix, and $\mathbf{M}_{\mathbf{u}}^{(i)}=4 \mathbf{E}^{(i)}{ }^{\mathbf{T}} \hat{\mathbf{J}}^{(i)} \mathbf{E}^{(i)}$ is a positive definite $4 \times 4$ matrix. Then Eq. (29) describes the orbital and attitude dynamics of the $i$ th follower satellite when no constraint (control) is applied to the system.

Let $\mathbf{F}^{\mathbf{C}(i)}(t)$ be the control force three vector for orbital control and $\Gamma_{\mathbf{u}}^{\mathbf{C}(i)}(t)$ be the generalized quaternion torque four vector for attitude control, then they form the constraint (control) force seven vector $\mathbf{Q}^{\mathbf{C}(i)}(t)$ in Eq. (11), that is,

$$
\mathbf{Q}^{\mathbf{C}(i)}(t)=\left[\begin{array}{l}
\mathbf{F}^{\mathbf{C}(i)}(t) \\
\boldsymbol{\Gamma}_{\mathbf{u}}^{\mathbf{C}(i)}(t)
\end{array}\right]
$$

When constraints on either orbital and/or attitude motion, which are of the form of Eq. (7), are applied, the constraint force and torque $\mathbf{Q}^{\mathbf{C}(i)}(t)$ required to follow the given constrained trajectory are explicitly determined by Eq. (11). In addition, one can relate 
the generalized quaternion torque four vector $\boldsymbol{\Gamma}_{\mathbf{u}}^{\mathbf{C}(i)}$ to the physically applied torque three vector about the body axis, $\boldsymbol{\Gamma}^{\mathbf{C}(i)}=\left[\begin{array}{lll}\Gamma_{x}^{(i)} & \Gamma_{y}^{(i)} & \Gamma_{z}^{(i)}\end{array}\right]^{\mathbf{T}}$, through the relation [37]

$$
\boldsymbol{\Gamma}^{\mathbf{C}(i)}=\frac{1}{2} \mathbf{E}_{1} \boldsymbol{\Gamma}_{\mathbf{u}}^{\mathbf{C}(i)}, \quad i=1,2, \cdots, N
$$

where $\mathbf{E}_{1}$ is defined in Eq. (21). The methodology presented earlier can be more easily explained by taking a specific example, as will be shown in the next subsection.

\section{Determining the Explicit Form of the Control Forces and Torques}

With the aid of the analytical expression for the control forces $\left[\mathbf{F}^{\mathbf{C}(i)}(t)\right]$ and torques $\left[\boldsymbol{\Gamma}_{\mathbf{u}}^{\mathbf{C}(i)}(t)\right]$ given by Eq. $(11)$, it is possible to obtain these control functions in closed form once orbital and/or attitude constraints are given. These analytical solutions provide many important physical insights into the dynamics of multiple satellites, which cannot be easily inferred from numerical computations, and which will be shown in the next section. The methodology adopted in this paper is general, in the sense that the explicit form of the control forces and torques is immediately obtained once constraints are given. Now, the following three kinds of constraints are considered.

Let us first assume that, for the $i$ th follower satellite, the following form of the constraints is obtained from $s$ consistent orbital constraints:

$$
\mathbf{A}_{11}(\mathbf{X}, \dot{\mathbf{X}}, t) \ddot{\mathbf{X}}=\mathbf{b}_{1}(\mathbf{X}, \dot{\mathbf{X}}, t)
$$

where $\mathbf{A}_{11}$ is an $s \times 3$ matrix, $\ddot{\mathbf{X}}=\left[\begin{array}{lll}\ddot{X} & \ddot{Y} & \ddot{Z}\end{array}\right]^{\mathbf{T}}$ is the acceleration three vector of the $i$ th follower satellite in the ECI frame, $\mathbf{b}_{1}$ is an $s$ vector, and $s(\leq 3)$ is the number of the given (independent) orbital constraints. Also, as in Eq. (33), the superscript $i$ will be omitted for brevity from here on, unless required for clarity.

As a specific example, let us consider the PCO [7, $, 2,38]$. Let the $i$ th follower satellite be required to have a PCO expressed in the Hill frame as

$$
\begin{gathered}
y^{2}+z^{2}=\rho_{0}^{2} \\
2 x=z
\end{gathered}
$$

where there are two constraints, and so $s$ is 2. Equation (34a) states that the follower satellite should move on a circle with constant radius $\rho_{0}$, where $\rho_{0}$ is the constant distance between the leader satellite and

$$
\begin{gathered}
\left(\mathbf{R}_{2} \mathbf{X}\right)^{2}+\left(\mathbf{R}_{3} \mathbf{X}\right)^{2}=\rho_{0}^{2} \\
2\left(\mathbf{R}_{1} \mathbf{X}-r_{L}\right)=\mathbf{R}_{3} \mathbf{X}
\end{gathered}
$$

where $\mathbf{R}_{1}, \mathbf{R}_{2}$, and $\mathbf{R}_{3}$ are the first, second, and third row vectors of the rotation matrix $\mathbf{R}$ in Eq. (16), respectively. To obtain a general form of the constraint equation expressed by Eq. (8), the following are defined:

$$
\begin{gathered}
\varphi_{1}:=\left(\mathbf{R}_{2} \mathbf{X}\right)^{2}+\left(\mathbf{R}_{3} \mathbf{X}\right)^{2}-\rho_{0}^{2} \\
\varphi_{2}:=2\left(\mathbf{R}_{1} \mathbf{X}-r_{L}\right)-\mathbf{R}_{3} \mathbf{X}
\end{gathered}
$$

so that

$$
\begin{gathered}
\dot{\varphi}_{1}=2\left(\mathbf{R}_{2} \mathbf{X}\right)\left(\dot{\mathbf{R}}_{2} \mathbf{X}+\mathbf{R}_{2} \dot{\mathbf{X}}\right)+2\left(\mathbf{R}_{3} \mathbf{X}\right)\left(\dot{\mathbf{R}}_{3} \mathbf{X}+\mathbf{R}_{3} \dot{\mathbf{X}}\right) \\
\dot{\varphi}_{2}=2\left(\dot{\mathbf{R}}_{1} \mathbf{X}+\mathbf{R}_{1} \dot{\mathbf{X}}-\dot{r}_{L}\right)-\left(\dot{\mathbf{R}}_{3} \mathbf{X}+\mathbf{R}_{3} \dot{\mathbf{X}}\right)
\end{gathered}
$$

and

$$
\begin{aligned}
\ddot{\varphi}_{1} & =2\left(\dot{\mathbf{R}}_{2} \mathbf{X}+\mathbf{R}_{2} \dot{\mathbf{X}}\right)^{2}+2\left(\mathbf{R}_{2} \mathbf{X}\right)\left(\ddot{\mathbf{R}}_{2} \mathbf{X}+2 \dot{\mathbf{R}}_{2} \dot{\mathbf{X}}+\mathbf{R}_{2} \ddot{\mathbf{X}}\right) \\
& +2\left(\dot{\mathbf{R}}_{3} \mathbf{X}+\mathbf{R}_{3} \dot{\mathbf{X}}\right)^{2}+2\left(\mathbf{R}_{3} \mathbf{X}\right)\left(\ddot{\mathbf{R}}_{3} \mathbf{X}+2 \dot{\mathbf{R}}_{3} \dot{\mathbf{X}}+\mathbf{R}_{3} \ddot{\mathbf{X}}\right)
\end{aligned}
$$

$$
\ddot{\varphi}_{2}=2\left(\ddot{\mathbf{R}}_{1} \mathbf{X}+2 \dot{\mathbf{R}}_{1} \dot{\mathbf{X}}+\mathbf{R}_{1} \ddot{\mathbf{X}}-\ddot{r}_{L}\right)-\left(\ddot{\mathbf{R}}_{3} \mathbf{X}+2 \dot{\mathbf{R}}_{3} \dot{\mathbf{X}}+\mathbf{R}_{3} \ddot{\mathbf{X}}\right)
$$

Then, using Eq. (8), one can obtain the constraint equations in the form of Eq. (7):

$$
\begin{gathered}
2\left(\left(\mathbf{R}_{2} \mathbf{X}\right) \mathbf{R}_{2}+\left(\mathbf{R}_{3} \mathbf{X}\right) \mathbf{R}_{3}\right) \ddot{\mathbf{X}}=-2\left(\dot{\mathbf{R}}_{2} \mathbf{X}+\mathbf{R}_{2} \dot{\mathbf{X}}\right)^{2}-2\left(\mathbf{R}_{2} \mathbf{X}\right) \\
\times\left(\ddot{\mathbf{R}}_{2} \mathbf{X}+2 \dot{\mathbf{R}}_{2} \dot{\mathbf{X}}\right)-2\left(\dot{\mathbf{R}}_{3} \mathbf{X}+\mathbf{R}_{3} \dot{\mathbf{X}}\right)^{2} \\
-2\left(\mathbf{R}_{3} \mathbf{X}\right)\left(\ddot{\mathbf{R}}_{3} \mathbf{X}+2 \dot{\mathbf{R}}_{3} \dot{\mathbf{X}}\right)-\alpha_{1} \dot{\varphi}_{1}-\beta_{1} \varphi_{1} \\
\left(2 \mathbf{R}_{1}-\mathbf{R}_{3}\right) \ddot{\mathbf{X}}=2 \ddot{r}_{L}-2\left(\ddot{\mathbf{R}}_{1} \mathbf{X}+2 \dot{\mathbf{R}}_{1} \dot{\mathbf{X}}\right) \\
+\left(\ddot{\mathbf{R}}_{3} \mathbf{X}+2 \dot{\mathbf{R}}_{3} \dot{\mathbf{X}}\right)-\alpha_{2} \dot{\varphi}_{2}-\beta_{2} \varphi_{2}
\end{gathered}
$$

Equation (39) can be compactly written as

$$
\left[\begin{array}{c}
2\left(\left(\mathbf{R}_{2} \mathbf{X}\right) \mathbf{R}_{2}+\left(\mathbf{R}_{3} \mathbf{X}\right) \mathbf{R}_{3}\right) \\
2 \mathbf{R}_{1}-\mathbf{R}_{3}
\end{array}\right] \ddot{\mathbf{X}}=\left[\begin{array}{c}
-2\left(\dot{\mathbf{R}}_{2} \mathbf{X}+\mathbf{R}_{2} \dot{\mathbf{X}}\right)^{2}-2\left(\mathbf{R}_{2} \mathbf{X}\right)\left(\ddot{\mathbf{R}}_{2} \mathbf{X}+2 \dot{\mathbf{R}}_{2} \dot{\mathbf{X}}\right)-2\left(\dot{\mathbf{R}}_{3} \mathbf{X}+\mathbf{R}_{3} \dot{\mathbf{X}}\right)^{2} \\
-2\left(\mathbf{R}_{3} \mathbf{X}\right)\left(\ddot{\mathbf{R}}_{3} \mathbf{X}+2 \dot{\mathbf{R}}_{3} \dot{\mathbf{X}}\right)-\alpha_{1} \dot{\varphi}_{1}-\beta_{1} \varphi_{1} \\
2 \ddot{r}_{L}-2\left(\ddot{\mathbf{R}}_{1} \mathbf{X}+2 \dot{\mathbf{R}}_{1} \dot{\mathbf{X}}\right)+\left(\ddot{\mathbf{R}}_{3} \mathbf{X}+2 \dot{\mathbf{R}}_{3} \dot{\mathbf{X}}\right)-\alpha_{2} \dot{\varphi}_{2}-\beta_{2} \varphi_{2}
\end{array}\right]
$$

which is of the form of Eq. (33) with

$$
\begin{gathered}
\mathbf{A}_{11}(\mathbf{X}, \dot{\mathbf{X}}, t)=\left[\begin{array}{c}
2\left(\left(\mathbf{R}_{2} \mathbf{X}\right) \mathbf{R}_{2}+\left(\mathbf{R}_{3} \mathbf{X}\right) \mathbf{R}_{3}\right) \\
2 \mathbf{R}_{1}-\mathbf{R}_{3}
\end{array}\right] \\
\mathbf{b}_{1}(\mathbf{X}, \dot{\mathbf{X}}, t)=\left[\begin{array}{c}
-2\left(\dot{\mathbf{R}}_{2} \mathbf{X}+\mathbf{R}_{2} \dot{\mathbf{X}}\right)^{2}-2\left(\mathbf{R}_{2} \mathbf{X}\right)\left(\ddot{\mathbf{R}}_{2} \mathbf{X}+2 \dot{\mathbf{R}}_{2} \dot{\mathbf{X}}\right)-2\left(\dot{\mathbf{R}}_{3} \mathbf{X}+\mathbf{R}_{3} \dot{\mathbf{X}}\right)^{2} \\
-2\left(\mathbf{R}_{3} \mathbf{X}\right)\left(\ddot{\mathbf{R}}_{3} \mathbf{X}+2 \dot{\mathbf{R}}_{3} \dot{\mathbf{X}}\right)-\alpha_{1} \dot{\varphi}_{1}-\beta_{1} \varphi_{1} \\
2 \ddot{r}_{L}-2\left(\ddot{\mathbf{R}}_{1} \mathbf{X}+2 \dot{\mathbf{R}}_{1} \dot{\mathbf{X}}\right)+\left(\ddot{\mathbf{R}}_{3} \mathbf{X}+2 \dot{\mathbf{R}}_{3} \dot{\mathbf{X}}\right)-\alpha_{2} \dot{\varphi}_{2}-\beta_{2} \varphi_{2}
\end{array}\right]
\end{gathered}
$$

the $i$ th follower satellite when projected on the local horizontal $(y-z)$ plane corresponding to it. Equation (35b) makes the relative motion bounded for every axis, and it also matches the solutions of the HCW equations satisfying the constraints Eq. (34a) [38]. Each follower satellite can have its own orbital requirements with respect to the leader satellite that can be correspondingly expressed as, for example, in Eq. (34). By transforming Eq. (34) to the ECI frame, the two orbital constraints can be expressed as where $\mathbf{A}_{11}$ is a $2 \times 3$ matrix and $\mathbf{b}_{1}$ is a two-vector. It is noted in passing that the matrix $\mathbf{A}_{11}$ and the vector $\mathbf{b}_{1}$ do not involve any quaternions. As with Eq. (34), it shall be assumed from here on that the $s(\leq 3)$ orbital constraints for each of the follower satellites are independent of one another, so that the rank of the matrix $\mathbf{A}_{11}$ is full.

Next, let us consider attitude constraints for target tracking. It is assumed that the $z$ axis of the body frame of the $i$ th follower satellite 
described by the unit vector $\hat{\mathbf{z}}_{\text {body }}^{(i)}=\left[\begin{array}{lll}0 & 0 & 1\end{array}\right]^{\mathbf{T}}$ is required to point to a given target and that the target's position vector in the ECI frame, which is given by $\mathbf{X}_{\mathbf{t}}^{(i)}(t)=\left[\begin{array}{lll}X_{t}^{(i)}(t) & Y_{t}^{(i)}(t) & Z_{t}^{(i)}(t)\end{array}\right]^{\mathbf{T}}$, may be an arbitrarily prescribed time-varying function. This constraint can be mathematically expressed using the fact that, in the body frame of reference, the vector $\hat{\mathbf{z}}_{\text {body }}^{(i)}$ should be parallel to the vector $\boldsymbol{\xi}^{(i)}(t):=\mathbf{X}_{\mathbf{t}}^{(i)}(t)-\mathbf{X}^{(i)}(t)=$ $\left[X_{t}^{(i)}(t)-X^{(i)}(t) \quad Y_{t}^{(i)}(t)-Y^{(i)}(t) \quad Z_{t}^{(i)}(t)-Z^{(i)}(t)\right]^{\mathbf{T}} \neq 0 \quad$ connecting the follower satellite and the target. As before, the superscript $i$ shall be dropped in what follows, for brevity, but note that each follower satellite is required to track, in general, a different target whose position may be arbitrarily prescribed in time. The aim is to perform, along with precise orbital control, attitude control, so that each follower satellite can precisely track its assigned target.

Let $\mathbf{T}$ be the transformation matrix that maps the ECI frame into the body frame of the $i$ th follower satellite, which is of the form

$$
\begin{aligned}
\mathbf{T} & :=\left[\begin{array}{l}
\mathbf{T}_{1} \\
\mathbf{T}_{2} \\
\mathbf{T}_{3}
\end{array}\right]:=\left[\begin{array}{lll}
T_{11} & T_{12} & T_{13} \\
T_{21} & T_{22} & T_{23} \\
T_{31} & T_{32} & T_{33}
\end{array}\right] \\
& =\left[\begin{array}{ccc}
u_{0}^{2}+u_{1}^{2}-u_{2}^{2}-u_{3}^{2} & 2\left(u_{1} u_{2}+u_{0} u_{3}\right) & 2\left(u_{1} u_{3}-u_{0} u_{2}\right) \\
2\left(u_{1} u_{2}-u_{0} u_{3}\right) & u_{0}^{2}-u_{1}^{2}+u_{2}^{2}-u_{3}^{2} & 2\left(u_{2} u_{3}+u_{0} u_{1}\right) \\
2\left(u_{1} u_{3}+u_{0} u_{2}\right) & 2\left(u_{2} u_{3}-u_{0} u_{1}\right) & u_{0}^{2}-u_{1}^{2}-u_{2}^{2}+u_{3}^{2}
\end{array}\right]
\end{aligned}
$$

where $\mathbf{T}_{1}, \mathbf{T}_{2}$, and $\mathbf{T}_{3}$ are the first, second, and third row vectors of the $\mathbf{T}$ matrix, respectively. Then, $\mathbf{T} \boldsymbol{\xi}(t)$ is the vector connecting the follower satellite and the target expressed along the body frame, and the attitude constraint for target pointing becomes

$$
\hat{\mathbf{z}}_{\text {body }} \times \mathbf{T} \boldsymbol{\xi}:=\hat{\mathbf{z}}_{\text {body }} \times\left[\begin{array}{c}
\mathbf{T}_{1} \boldsymbol{\xi} \\
\mathbf{T}_{2} \boldsymbol{\xi} \\
\mathbf{T}_{3} \boldsymbol{\xi}
\end{array}\right]=0
$$

Equation (43) describes the attitude tracking requirement for the $i$ th follower satellite, and each of the $N$ follower satellites can have their own time-varying attitude requirements for tracking different targets.

Expanding Eq. (43) for the $i$ th follower satellite, one has

$$
\begin{aligned}
& {\left[\begin{array}{ccc}
0 & -1 & 0 \\
1 & 0 & 0 \\
0 & 0 & 0
\end{array}\right]\left[\begin{array}{c}
\mathbf{T}_{1} \boldsymbol{\xi} \\
\mathbf{T}_{2} \boldsymbol{\xi} \\
\mathbf{T}_{3} \boldsymbol{\xi}
\end{array}\right]=\left[\begin{array}{ccc}
0 & -1 & 0 \\
1 & 0 & 0 \\
0 & 0 & 0
\end{array}\right]} \\
& \times\left[\begin{array}{c}
T_{11}\left(X_{t}-X\right)+T_{12}\left(Y_{t}-Y\right)+T_{13}\left(Z_{t}-Z\right) \\
T_{21}\left(X_{t}-X\right)+T_{22}\left(Y_{t}-Y\right)+T_{23}\left(Z_{t}-Z\right) \\
T_{31}\left(X_{t}-X\right)+T_{32}\left(Y_{t}-Y\right)+T_{33}\left(Z_{t}-Z\right)
\end{array}\right]=\left[\begin{array}{l}
0 \\
0 \\
0
\end{array}\right]
\end{aligned}
$$

Then, the two constraints for target pointing are given by

$$
\begin{gathered}
-\left[T_{21}\left(X_{t}-X\right)+T_{22}\left(Y_{t}-Y\right)+T_{23}\left(Z_{t}-Z\right)\right]=0 \\
T_{11}\left(X_{t}-X\right)+T_{12}\left(Y_{t}-Y\right)+T_{13}\left(Z_{t}-Z\right)=0
\end{gathered}
$$

Equations ( $\underline{45 \mathrm{a}})$ and $(\underline{45 \mathrm{~b}})$ can also be compactly written, for future use, as

$$
\begin{gathered}
-\mathbf{T}_{2} \boldsymbol{\xi}=0 \\
\mathbf{T}_{1} \boldsymbol{\xi}=0
\end{gathered}
$$

As before, the following are defined:

$$
\begin{array}{cc}
\varphi_{3}:=-\mathbf{T}_{2} \xi, & \dot{\varphi}_{3}:=-\dot{\mathbf{T}}_{2} \boldsymbol{\xi}-\mathbf{T}_{2} \dot{\xi} \\
\varphi_{4}:=\mathbf{T}_{1} \xi, & \dot{\varphi}_{4}=\dot{\mathbf{T}}_{1} \boldsymbol{\xi}+\mathbf{T}_{1} \dot{\xi}
\end{array}
$$

Besides these two requirements, it is necessary to impose an additional constraint that the quaternion four vector of each follower satellite must have unit norm, so that physical rotations are described. This constraint is of the form

$$
N(\mathbf{u}):=u_{0}^{2}+u_{1}^{2}+u_{2}^{2}+u_{3}^{2}=1
$$

so that

$$
\begin{aligned}
& \varphi_{5}:=u_{0}^{2}+u_{1}^{2}+u_{2}^{2}+u_{3}^{2}-1 \\
& \dot{\varphi}_{5}=2\left(u_{0} \dot{u}_{0}+u_{1} \dot{u}_{1}+u_{2} \dot{u}_{2}+u_{3} \dot{u}_{3}\right)
\end{aligned}
$$

Following the same procedure in the orbital part, one can have the following attitude constraint equations:

$$
\begin{gathered}
{\left[\begin{array}{ll}
\mathbf{A}_{21} & \mathbf{A}_{22}
\end{array}\right]\left[\begin{array}{c}
\ddot{\mathbf{X}} \\
\ddot{\mathbf{u}}
\end{array}\right]=\mathbf{b}_{2}} \\
\mathbf{A}_{21}(\mathbf{u})=\left[\begin{array}{ccc}
T_{21} & T_{22} & T_{23} \\
-T_{11} & -T_{12} & -T_{13} \\
0 & 0 & 0
\end{array}\right]:=\left[\begin{array}{c}
\overline{\mathbf{A}}_{21}(\mathbf{u}) \\
0_{1 \times 3}
\end{array}\right]
\end{gathered}
$$

\section{$\mathbf{A}_{22}\left(\mathbf{X}, \mathbf{X}_{\mathbf{t}}, \mathbf{u}\right)$}

$$
\begin{aligned}
2\left[\begin{array}{lll}
u_{3} & -u_{0} & -u_{1}
\end{array}\right]\left[\begin{array}{c}
X_{t}-X \\
Y_{t}-Y \\
Z_{t}-Z
\end{array}\right] & 2\left[\begin{array}{lll}
-u_{2} & u_{1} & -u_{0}
\end{array}\right]\left[\begin{array}{c}
X_{t}-X \\
Y_{t}-Y \\
Z_{t}-Z
\end{array}\right] \\
2\left[\begin{array}{lll}
u_{0} & u_{3} & -u_{2}
\end{array}\right]\left[\begin{array}{l}
X_{t}-X \\
Y_{t}-Y \\
Z_{t}-Z
\end{array}\right] & 2\left[\begin{array}{lll}
u_{1} & u_{2} & u_{3}
\end{array}\right]\left[\begin{array}{c}
X_{t}-X \\
Y_{t}-Y \\
Z_{t}-Z
\end{array}\right] \\
:= & {\left[\begin{array}{c}
\mathbf{A}_{22}\left(\mathbf{X}, \mathbf{X}_{\mathbf{t}}, \mathbf{u}\right) \\
2 u^{\mathbf{T}}
\end{array}\right] }
\end{aligned}
$$$$
\begin{gathered}
2\left[\begin{array}{lll}
-u_{1} & -u_{2} & -u_{3}
\end{array}\right]\left[\begin{array}{c}
X_{t}-X \\
Y_{t}-Y \\
Z_{t}-Z
\end{array}\right] \\
2\left[\begin{array}{lll}
-u_{2} & u_{1} & -u_{0}
\end{array}\right]\left[\begin{array}{c}
X_{t}-X \\
Y_{t}-Y \\
Z_{t}-Z
\end{array}\right]
\end{gathered}
$$$$
2 u_{2}
$$

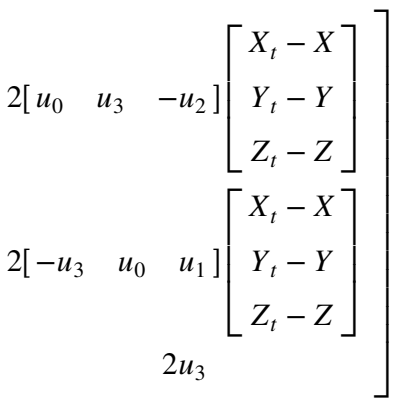




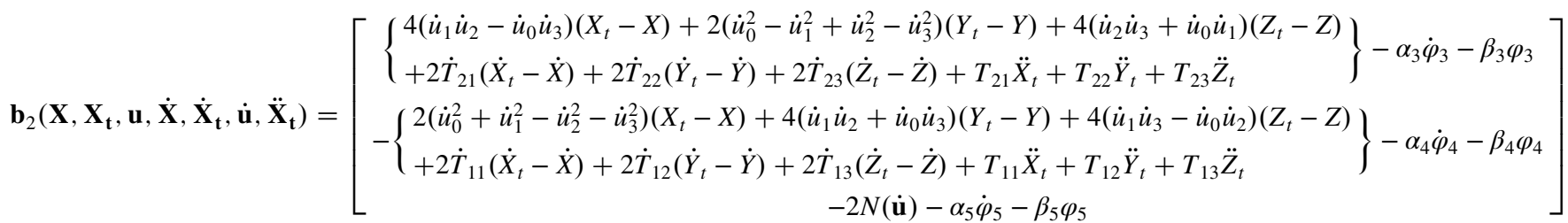

$$
\begin{aligned}
& :=\left[\begin{array}{c}
\overline{\mathbf{b}}_{2}\left(\mathbf{X}, \mathbf{X}_{\mathbf{t}}, \mathbf{u}, \dot{\mathbf{X}}, \dot{\mathbf{X}}_{\mathbf{t}}, \dot{\mathbf{u}}, \ddot{\mathbf{X}}_{\mathbf{t}}\right) \\
-2 N(\dot{\mathbf{u}})-\alpha_{5} \dot{\varphi}_{5}-\beta_{5} \varphi_{5}
\end{array}\right]
\end{aligned}
$$

where $N(\dot{\mathbf{u}}):=\dot{u}_{0}^{2}+\dot{u}_{1}^{2}+\dot{u}_{2}^{2}+\dot{u}_{3}^{2}$. The matrix $\mathbf{A}_{21}$ is a $3 \times 3$ matrix, $\mathbf{A}_{22}$ is a $3 \times 4$ matrix, and $\mathbf{b}_{2}$ is a three vector; to obtain the corresponding barred quantities, the last row in each of these matrices needs to be dropped. In Appendix $\underline{\mathrm{A}}$, it is shown that $\mathbf{A}_{22}$ always has full rank.

Finally, the constraint equation, which is of the form of Eq. (7), including both orbital and attitude constraints, is written as

$$
\mathbf{A} \ddot{\mathbf{q}}:=\left[\begin{array}{cc}
\mathbf{A}_{11} & 0 \\
\mathbf{A}_{21} & \mathbf{A}_{22}
\end{array}\right]\left[\begin{array}{l}
\ddot{\mathbf{X}} \\
\ddot{\mathbf{u}}
\end{array}\right]:=\left[\begin{array}{l}
\mathbf{b}_{1} \\
\mathbf{b}_{2}
\end{array}\right]:=\mathbf{b}
$$

where the matrix $\mathbf{A}$ is, in general, $(s+3) \times 7)$ and $s \leq 3$ is the number of independent orbital constraints or the number of the independent rows of the matrix $\mathbf{A}_{11}$. Then, the control force and torque are explicitly calculated using Eqs. (11a) or (11b). In Appendix A, it is proved that the matrix A in Eq. (53) always has full rank. Then, one can use the regular inverse instead of the MPgeneralized inverse of the matrix $\mathbf{A} \mathbf{M}^{-1} \mathbf{A}^{\mathbf{T}}$ in Eq. (11b), so that the control forces $\left[\mathbf{F}^{\mathbf{C}}(t)\right]$ and torques $\left[\boldsymbol{\Gamma}_{\mathbf{u}}^{\mathbf{C}}(t)\right]$ on the $i$ th follower satellite are given explicitly by the seven vector

$$
\mathbf{Q}^{\mathbf{C}}(t):=\left[\begin{array}{l}
\mathbf{F}^{\mathbf{C}}(t) \\
\boldsymbol{\Gamma}_{u}^{C}(t)
\end{array}\right]=\mathbf{A}^{\mathbf{T}}\left(\mathbf{A} \mathbf{M}^{-1} \mathbf{A}^{\mathbf{T}}\right)^{-1}(\mathbf{b}-\mathbf{A} \mathbf{a})
$$

Using the regular inverse as in Eq. (54), instead of the MPgeneralized inverse as in Eq. (11b), saves considerable computation time and dramatically improves numerical accuracy. The first component of $\mathbf{Q}^{\mathbf{C}}(t)$, which is a three vector, $\mathbf{F}^{\mathbf{C}}(t)$, is the control force for orbital control, whereas the second component, which is a four vector, $\boldsymbol{\Gamma}_{u}^{\mathbf{C}}(t)$, is the generalized control torque for attitude control. In Appendix B, it is shown that the right-hand side of Eq. (54) simplifies to yield

$$
\begin{aligned}
\mathbf{F}^{\mathbf{C}}(t) & =m \alpha_{\mathbf{O}}+m \mathbf{P}_{1} \mathbf{A}_{21}^{\mathbf{T}} \mathbf{D}\left(\mathbf{b}_{2}-\mathbf{A}_{21} \mathbf{a}_{1}-\mathbf{A}_{22} \mathbf{a}_{2}-\mathbf{A}_{21} \boldsymbol{\alpha}_{\mathbf{O}}\right) \\
& =m \alpha_{\mathbf{O}}+m \mathbf{P}_{1} \mathbf{A}_{21}^{\mathbf{T}} \alpha_{\mathbf{O}, \mathbf{A}}
\end{aligned}
$$

$$
\boldsymbol{\Gamma}_{u}^{\mathbf{C}}(t)=m \mathbf{A}_{22}^{\mathbf{T}} \mathbf{D}\left(\mathbf{b}_{2}-\mathbf{A}_{21} \mathbf{a}_{1}-\mathbf{A}_{22} \mathbf{a}_{2}-\mathbf{A}_{21} \boldsymbol{\alpha}_{\mathbf{O}}\right)=m \mathbf{A}_{22}^{\mathbf{T}} \alpha_{\mathbf{O}, \mathbf{A}}
$$

where

$$
\begin{aligned}
& \mathbf{P}_{0}(\mathbf{X}, \dot{\mathbf{X}}, t):=\mathbf{A}_{11}^{\mathbf{T}}\left(\mathbf{A}_{11} \mathbf{A}_{11}^{\mathbf{T}}\right)^{-1} \mathbf{A}_{11}=\mathbf{A}_{11}^{+} \mathbf{A}_{11} \\
& \mathbf{P}_{1}(\mathbf{X}, \dot{\mathbf{X}}, t):=\mathbf{I}_{3 \times 3}-\mathbf{P}_{0}(\mathbf{X}, \dot{\mathbf{X}}, t)=\mathbf{I}_{3 \times 3}-\mathbf{A}_{11}^{\mathbf{T}}\left(\mathbf{A}_{11} \mathbf{A}_{11}^{\mathbf{T}}\right)^{-1} \mathbf{A}_{11} \\
& \quad=\mathbf{I}_{3 \times 3}-\mathbf{A}_{11}^{+} \mathbf{A}_{11} \\
& \boldsymbol{\alpha}_{\mathbf{O}}(\mathbf{X}, \dot{\mathbf{X}}, t):=\mathbf{A}_{11}^{\mathbf{T}}\left(\mathbf{A}_{11} \mathbf{A}_{11}^{\mathbf{T}}\right)^{-1}\left(\mathbf{b}_{1}-\mathbf{A}_{11} \mathbf{a}_{1}\right)=\mathbf{A}_{11}^{+}\left(\mathbf{b}_{1}-\mathbf{A}_{11} \mathbf{a}_{1}\right) \\
& \mathbf{D}\left(\mathbf{X}, \mathbf{X}_{\mathbf{t}}, \mathbf{u}, \dot{\mathbf{X}}, t\right):=\left(\mathbf{A}_{21} \mathbf{P}_{1} \mathbf{A}_{21}^{\mathbf{T}}+m \mathbf{A}_{22} \mathbf{M}_{\mathbf{u}}^{-1} \mathbf{A}_{22}^{\mathbf{T}}\right)^{-1}
\end{aligned}
$$

and

$$
\begin{aligned}
& \boldsymbol{\alpha}_{\mathbf{O}, \mathbf{A}}\left(\mathbf{X}, \mathbf{X}_{\mathbf{t}}, \mathbf{u}, \dot{\mathbf{X}}, \dot{\mathbf{X}}_{\mathbf{t}}, \dot{\mathbf{u}}, \ddot{\mathbf{X}}_{\mathbf{t}}, t\right) \\
& \quad:=\mathbf{D}\left(\mathbf{b}_{2}-\mathbf{A}_{21} \mathbf{a}_{1}-\mathbf{A}_{22} \mathbf{a}_{2}-\mathbf{A}_{21} \boldsymbol{\alpha}_{\mathbf{O}}\right)
\end{aligned}
$$

The matrices $\mathbf{P}_{0}$ and $\mathbf{P}_{1}$ are orthogonal projection operators because they are both symmetric idempotent matrices. The matrix $\mathbf{P}_{0}$ is a projection onto the column space of $\mathbf{A}_{11}^{\mathbf{T}}$, and the matrix $\mathbf{P}_{1}$ is the projection onto the null space of $\mathbf{A}_{11}$. It should be noted that the matrix $\mathbf{D}$ in Eq. (58) is well defined because the $3 \times 4$ matrix $\mathbf{A}_{22}$ has full rank (see Appendix A), so that $\mathbf{A}_{22} \mathbf{M}_{\mathbf{u}}^{-1} \mathbf{A}_{22}^{\mathbf{T}}$ is positive definite, and $\mathbf{A}_{21} \mathbf{P}_{1} \mathbf{A}_{21}^{\mathbf{T}}$ is semipositive definite because $\mathbf{P}_{1}$ is idempotent. Let us briefly investigate the relationship between $\mathbf{P}_{0}$ and $\mathbf{P}_{1}$. First, it should be pointed out that [39]

$$
\operatorname{rank}\left(\mathbf{P}_{0}\right)=\operatorname{rank}\left(\mathbf{A}_{11}^{+} \mathbf{A}_{11}\right)=\operatorname{rank}\left(\mathbf{A}_{11}\right)=s
$$

Because the column space of $\mathbf{P}_{1}=\mathbf{I}_{3 \times 3}-\mathbf{A}_{11}^{+} \mathbf{A}_{11}$ is the same as the null space of $\mathbf{A}_{11}$ [39], the rank of $\mathbf{P}_{1}$ is

$$
\operatorname{rank}\left(\mathbf{P}_{1}\right)=\operatorname{nullity}\left(\mathbf{A}_{11}\right)=3-s
$$

Combining Eqs. (60) and (다) yields

$$
\operatorname{rank}\left(\mathbf{P}_{0}\right)+\operatorname{rank}\left(\mathbf{P}_{1}\right)=3
$$

and Eqs. (61) and (62) will be used later.

The structure of Eqs. (55) and (56) is better exposed when written in the form

$$
\left[\begin{array}{l}
\mathbf{F}^{\mathbf{C}}(t) \\
\boldsymbol{\Gamma}^{\mathbf{C}}(t)
\end{array}\right]=m\left[\begin{array}{cc}
\mathbf{I}_{3 \times 3} & \mathbf{P}_{1} \mathbf{A}_{21}^{\mathbf{T}} \\
0 & \frac{1}{2} \mathbf{E}_{1} \mathbf{A}_{22}^{\mathbf{T}}
\end{array}\right]\left[\begin{array}{c}
\boldsymbol{\alpha}_{\mathbf{O}} \\
\boldsymbol{\alpha}_{\mathbf{O}, \mathbf{A}}
\end{array}\right]
$$

where Eqs. (21) and (32) have been used in Eq. (63) to obtain the physical torque three vector $\Gamma^{\mathbf{C}}$ applied to the follower satellite. Equation (63) is valid whether or not there is sensor measurement noise that causes the initial conditions, both in orbit and attitude, of the follower satellites to be incorrect at the start of the maneuver.

One can further particularize the general result given in Eq. (63) when the attitude constraints $(\underline{45})$ and the unit-norm constraint $(\overline{47})$ are satisfied and there are no attitude sensor measurement errors. As pointed out earlier, the attitude constraints will be asymptotically satisfied in the presence of sensor measurement noise. Recalling that [see Eqs. (50-52)], if the attitude constraints and the unit-norm constraint are met,

$$
\mathbf{A}_{21}=\left[\begin{array}{c}
\overline{\mathbf{A}}_{21} \\
0_{1 \times 3}
\end{array}\right], \quad \mathbf{A}_{22}=\left[\begin{array}{l}
\overline{\mathbf{A}}_{22} \\
2 \mathbf{u}^{\mathbf{T}}
\end{array}\right], \quad \mathbf{b}_{2}=\left[\begin{array}{c}
\overline{\mathbf{b}}_{2} \\
-2 N(\dot{\mathbf{u}})
\end{array}\right]
$$

and one can find that the control force and the physically applied torque can be rewritten as (see Appendix $\underline{\text { C) }}$

$$
\begin{gathered}
\mathbf{F}^{\mathbf{C}}(t)=m \alpha_{\mathbf{O}}+m \mathbf{P}_{1} \overline{\mathbf{A}}_{21}^{\mathbf{T}} \overline{\boldsymbol{\alpha}}_{\mathbf{O}, \mathbf{A}} \\
\boldsymbol{\Gamma}^{\mathbf{C}}(t)=\frac{m}{2} \mathbf{E}_{1} \overline{\mathbf{A}}_{22}^{\mathbf{T}} \overline{\boldsymbol{\alpha}}_{\mathbf{O}, \mathbf{A}}
\end{gathered}
$$

where

$$
\begin{aligned}
& \overline{\mathbf{D}}\left(\mathbf{X}, \mathbf{X}_{\mathbf{t}}, \mathbf{u}, \dot{\mathbf{X}}, t\right)=\left(\overline{\mathbf{A}}_{21} \mathbf{P}_{1} \overline{\mathbf{A}}_{21}^{\mathbf{T}}+\frac{m}{4} \overline{\mathbf{A}}_{22} \mathbf{E}_{1}^{\mathbf{T}} \mathbf{J}^{-1} \mathbf{E}_{1} \overline{\mathbf{A}}_{22}^{\mathbf{T}}\right)^{-1} \\
& \overline{\boldsymbol{\alpha}}_{\mathbf{O}, \mathbf{A}}\left(\mathbf{X}, \mathbf{X}_{\mathbf{t}}, \mathbf{u}, \dot{\mathbf{X}}, \dot{\mathbf{X}}_{\mathbf{t}}, \dot{\mathbf{u}}, \ddot{\mathbf{X}}_{\mathbf{t}}, t\right) \\
& \quad=\overline{\mathbf{D}}\left(\overline{\mathbf{b}}_{2}-\overline{\mathbf{A}}_{21} \mathbf{a}_{1}-\overline{\mathbf{A}}_{22} \mathbf{a}_{2}-\overline{\mathbf{A}}_{21} \boldsymbol{\alpha}_{\mathbf{O}}\right)
\end{aligned}
$$


with $\mathbf{P}_{1}$ and $\boldsymbol{\alpha}_{\mathbf{O}}$ defined in Eqs. (무) and (마). Also, the invertibility of the matrix $\overline{\mathbf{D}}$ in Eq. (67) is guaranteed. Note that the matrix $\mathbf{A}_{22} \mathbf{M}_{\mathbf{u}}^{-1} \mathbf{A}_{22}^{\mathrm{T}}$ is positive definite where

$$
\begin{aligned}
\mathbf{A}_{22} \mathbf{M}_{\mathbf{u}}^{-1} \mathbf{A}_{22}^{\mathbf{T}} & =\frac{1}{4}\left[\begin{array}{l}
\overline{\mathbf{A}}_{22} \\
2 \mathbf{u}^{\mathbf{T}}
\end{array}\right]\left[\begin{array}{ll}
\mathbf{u} & \mathbf{E}_{1}^{\mathbf{T}}
\end{array}\right]\left[\begin{array}{cc}
\frac{1}{J_{0}} & 0 \\
0 & \mathbf{J}^{-1}
\end{array}\right]\left[\begin{array}{l}
\mathbf{u}^{\mathbf{T}} \\
\mathbf{E}_{1}
\end{array}\right]\left[\begin{array}{ll}
\overline{\mathbf{A}}_{22}^{\mathbf{T}} & 2 \mathbf{u}
\end{array}\right] \\
& =\frac{1}{4}\left[\begin{array}{l}
\overline{\mathbf{A}}_{22} \\
2 \mathbf{u}^{\mathbf{T}}
\end{array}\right]\left(\frac{1}{J_{0}} \mathbf{u u}^{\mathbf{T}}+\mathbf{E}_{1}^{\mathbf{T}} \mathbf{J}^{-1} \mathbf{E}_{1}\right)\left[\begin{array}{ll}
\overline{\mathbf{A}}_{22}^{\mathbf{T}} & 2 \mathbf{u}
\end{array}\right] \\
& =\frac{1}{4}\left[\begin{array}{cc}
\overline{\mathbf{A}}_{22} \mathbf{E}_{1}^{\mathbf{T}} \mathbf{J}^{-1} \mathbf{E}_{1} \overline{\mathbf{A}}_{22}^{\mathbf{T}} & 0 \\
0 & \frac{4}{J_{0}}
\end{array}\right]>0
\end{aligned}
$$

and Eqs. (19), (21), and (64), and the identity $\mathbf{E}_{1} \mathbf{u}=0$, have been used in deriving Eq. (68), assuming that the attitude constraints and the unit-norm constraint, Eqs. (45c), (45d), and (47), are satisfied, so that $\overline{\mathbf{A}}_{22} \mathbf{u}=0$ in Eq. (A3) of Appendix A. From Eq. (68), it is concluded that the matrix $\overline{\mathbf{A}}_{21} \mathbf{P}_{1} \overline{\mathbf{A}}_{21}^{\mathbf{T}}+(m / 4) \overline{\mathbf{A}}_{22} \mathbf{E}_{1}^{\mathbf{T}} \mathbf{J}^{-1} \overline{\mathbf{E}_{1}} \overline{\mathbf{A}}_{22}^{\mathbf{T}}$ is positive definite because $\overline{\mathbf{A}}_{22} \mathbf{E}_{1}^{\mathbf{T}} \mathbf{J}^{-1} \mathbf{E}_{1} \overline{\mathbf{A}}_{22}^{\mathbf{T}}$ is positive definite and $\overline{\mathbf{A}}_{21} \mathbf{P}_{1} \overline{\mathbf{A}}_{21}^{\mathbf{T}}$ is semipositive definite, so that $\overline{\mathbf{D}}$ [see Eq. (67)] is well defined. Equation (63) now reads

$$
\left[\begin{array}{c}
\mathbf{F}^{\mathbf{C}}(t) \\
\boldsymbol{\Gamma}^{\mathbf{C}}(t)
\end{array}\right]=m\left[\begin{array}{cc}
\mathbf{I}_{3 \times 3} & \mathbf{P}_{1} \overline{\mathbf{A}}_{21}^{\mathbf{T}} \\
0 & \frac{1}{2} \mathbf{E}_{1} \overline{\mathbf{A}}_{22}^{\mathrm{T}}
\end{array}\right]\left[\begin{array}{c}
\boldsymbol{\alpha}_{\mathbf{O}} \\
\overline{\boldsymbol{\alpha}}_{\mathbf{O}, \mathbf{A}}
\end{array}\right]
$$

where the barred quantities are given in Eqs. $(\underline{50-52}), \overline{\boldsymbol{\alpha}}_{\mathbf{O}, \mathbf{A}}$ is defined in Eq. (67), and $\mathbf{P}_{1}$ is defined in Eq. (57).

In conclusion, Eqs. (63) and (69), which explicitly give the control forces and control torques, form part of the central results of this paper. It is important to note that Eq. (63) is more general than Eq. (69), in that Eq. (63) is applicable to cases when the attitude constraints $(\underline{45 \mathrm{c})}$ and $(\underline{45 \mathrm{~d}})$ are not exactly satisfied, for example, when initial condition errors caused by sensor measurement noise are present. Equation (69) is simpler than Eq. (63), but it is obtained based on the assumption that the attitude constraints $(\underline{45 \mathrm{c})}$ and $(\underline{45 \mathrm{~d}})$ are satisfied throughout the maneuver (including at the time of insertion), though not necessarily the orbital constraints. More precisely, Eq. (69) requires the unit-norm constraint (47) as well. If this constraint is not satisfied, it is impossible to describe physical rotation; hence, the quaternions have unit norm at the initial time and are controlled to maintain this norm by the controller developed in this paper.

Also, the acceleration vector of the constrained (controlled) system is explicitly obtained from Eq. (주) as

$$
\begin{aligned}
\ddot{\mathbf{q}} & =\left[\begin{array}{l}
\ddot{\mathbf{X}} \\
\ddot{\mathbf{u}}
\end{array}\right] \\
& =\left[\begin{array}{l}
\mathbf{a}_{1} \\
\mathbf{a}_{2}
\end{array}\right]+m\left[\begin{array}{cc}
m \mathbf{I}_{3 \times 3} & 0 \\
0 & \mathbf{M}_{\mathbf{u}}
\end{array}\right]^{-1}\left[\begin{array}{cc}
\mathbf{I}_{3 \times 3} & \mathbf{P}_{1} \mathbf{A}_{21}^{\mathrm{T}} \\
0 & \mathbf{A}_{22}^{\mathbf{T}}
\end{array}\right]\left[\begin{array}{c}
\boldsymbol{\alpha}_{\mathbf{O}} \\
\boldsymbol{\alpha}_{\mathbf{O}, \mathbf{A}}
\end{array}\right] \\
& =\left[\begin{array}{l}
\mathbf{a}_{1} \\
\mathbf{a}_{2}
\end{array}\right]+\left[\begin{array}{cc}
\mathbf{I}_{3 \times 3} & \mathbf{P}_{1} \mathbf{A}_{21}^{\mathrm{T}} \\
0 & m \mathbf{M}_{\mathbf{u}}^{-1} \mathbf{A}_{22}^{\mathrm{T}}
\end{array}\right]\left[\begin{array}{c}
\boldsymbol{\alpha}_{\mathbf{O}} \\
\boldsymbol{\alpha}_{\mathbf{O}, \mathbf{A}}
\end{array}\right]
\end{aligned}
$$

or from Eq. (9) as

$$
\begin{aligned}
\ddot{\mathbf{q}} & =\left[\begin{array}{l}
\ddot{\mathbf{X}} \\
\ddot{\mathbf{u}}
\end{array}\right] \\
& =\left[\begin{array}{c}
\mathbf{a}_{1} \\
\mathbf{a}_{2}
\end{array}\right]+m\left[\begin{array}{cc}
m \mathbf{I}_{3 \times 3} & 0 \\
0 & \mathbf{M}_{\mathbf{u}}
\end{array}\right]^{-1}\left[\begin{array}{cc}
\mathbf{I}_{3 \times 3} & \mathbf{P}_{1} \overline{\mathbf{A}}_{21}^{\mathbf{T}} \\
0 & \overline{\mathbf{A}}_{22}^{\mathbf{T}}
\end{array}\right]\left[\begin{array}{c}
\boldsymbol{\alpha}_{\mathbf{O}} \\
\overline{\boldsymbol{\alpha}}_{\mathbf{O}, \mathbf{A}}
\end{array}\right] \\
& =\left[\begin{array}{c}
\mathbf{a}_{1} \\
\mathbf{a}_{2}
\end{array}\right]+\left[\begin{array}{cc}
\mathbf{I}_{3 \times 3} & \mathbf{P}_{1} \overline{\mathbf{A}}_{21}^{\mathrm{T}} \\
0 & m \mathbf{M}_{\mathbf{u}}^{-1} \overline{\mathbf{A}}_{22}^{\mathbf{T}}
\end{array}\right]\left[\begin{array}{c}
\boldsymbol{\alpha}_{\mathbf{O}} \\
\overline{\boldsymbol{\alpha}}_{\mathbf{O}, \mathbf{A}}
\end{array}\right]
\end{aligned}
$$

As before, Eq. (71) is restricted to situations when the attitude constraints (45c) and (45d) [and the unit-norm constraint (47)] are satisfied, Eq. (70) being the more general result. From Eqs. (58) and (59), it is seen that $\boldsymbol{\alpha}_{\mathbf{O}}$ is a function only of the orbital constraints (requirements), whereas $\boldsymbol{\alpha}_{\mathbf{O}, \mathbf{A}}$ depends on both the orbital and the attitude constraints (requirements). Thus, from Eq. (63), when $\mathbf{P}_{1} \mathbf{A}_{21}^{\mathbf{T}} \boldsymbol{\alpha}_{\mathbf{O}, \mathbf{A}}=0$, the control force $\mathbf{F}^{\mathbf{C}}(t)$ required to be applied to the follower satellite for orbital control does not depend on the attitude of the follower satellite or the attitude constraints it is required to satisfy. On the other hand, the control torques $\Gamma^{\mathrm{C}}(t)$ are dependent, in general, on both the orbital and the attitude requirements, and they depend on the orbital position and velocity of the follower satellite, and of course on $\dot{\mathbf{u}}$ and $\dot{\mathbf{u}}$. This leads to an important principle, explained in the next section.

\section{Separation Principle}

The structure of Eq. (63) leads to the following result.

When the independent orbital trajectory constraints (trajectory requirements) for the $i$ th follower are such that

$$
\mathbf{A}_{11}(\mathbf{X}, \dot{\mathbf{X}}, t) \ddot{\mathbf{X}}=\mathbf{b}_{1}(\mathbf{X}, \dot{\mathbf{X}}, t)
$$

where $\mathbf{X}(t)=\left[\begin{array}{lll}X(t) & Y(t) & Z(t)\end{array}\right]^{\mathbf{T}}$ is the position vector of the $i$ th follower satellite in the ECI frame, and the attitude pointing constraints (requirements) are such that

$$
\left[\begin{array}{ll}
\mathbf{A}_{21} & \mathbf{A}_{22}
\end{array}\right]\left[\begin{array}{c}
\ddot{\mathbf{X}} \\
\ddot{\mathbf{u}}
\end{array}\right]=\mathbf{b}_{2}
$$

where $\mathbf{u}$ is the quaternion four vector describing the orientation of the follower satellite, then the control force $\mathbf{F}^{\mathbf{C}}(t)$ on the follower satellite needed at time $t$ to satisfy the orbital constraints (requirements) will be independent of any attitude pointing constraints (requirements) at time $t$, provided at that time

$$
\mathbf{P}_{1} \mathbf{A}_{21}^{\mathbf{T}} \alpha_{\mathbf{O}, \mathbf{A}}=0
$$

is satisfied, where $\mathbf{P}_{1}$ and $\boldsymbol{\alpha}_{\mathbf{O}, \mathbf{A}}$ are, respectively, defined in Eqs. (57) and (59). The corresponding control force at time $t$ is given by $\mathbf{F}^{\mathbf{C}}(t)=m \alpha_{\mathbf{O}}$, where $\alpha_{\mathbf{O}}$ is explicitly given in Eq. (58), and it does not depend on the attitude of the follower satellite. Equation (74) can be often satisfied in one of two different ways, leading to the following two cases.

Case 1: When $\mathbf{P}_{1}=0$. For any controller that does exact orbital control, for all time, is independent of attitude control (or any attitude consideration) if $\mathbf{A}_{11}$ is invertible (i.e., if there are three independent orbital constraints). Only these three orbital constraints fully determine the orbital state vector $\mathbf{X}(t)$ and $\dot{\mathbf{X}}(t)$ with the given initial conditions. Regarding the controller employed in the current paper, when the matrix $\mathbf{A}_{11}$ is an invertible $3 \times 3$ matrix, $\mathbf{P}_{0}=\mathbf{I}_{3 \times 3}$, so that $\mathbf{P}_{1}=0$ and $\mathbf{P}_{1} \mathbf{A}_{21}^{\mathbf{T}} \boldsymbol{\alpha}_{\mathbf{O}, \mathbf{A}}=0$. The orbital control force will not then depend on the attitude of the follower satellite. More explicitly, it is obtained as

$$
\mathbf{F}^{\mathbf{C}}(t)=m\left(\mathbf{A}_{11}^{-1} \mathbf{b}_{1}-\mathbf{a}_{1}\right)
$$

Equation (75) is quite natural, in that $m \mathbf{A}_{11}^{-1} \mathbf{b}_{1}$ is the total force to satisfy the full orbital constraint and $m \mathbf{a}_{1}$ is just the force resulting from the unconstrained system, and so $m\left(\mathbf{A}_{11}^{-1} \mathbf{b}_{1}-\mathbf{a}_{1}\right)$ is the additional control force that is necessary to satisfy the given orbital constraints. Also, from Eq. $(\underline{58}), \mathbf{D}=(1 / m)\left(\mathbf{A}_{22} \mathbf{M}_{\mathbf{u}}^{-1} \mathbf{A}_{22}^{\mathrm{T}}\right)^{-1}$ and the attitude control torque is explicitly given by

$$
\begin{aligned}
\boldsymbol{\Gamma}^{\mathbf{C}}(t) & =\frac{1}{2} \mathbf{E}_{1} \mathbf{A}_{22}^{\mathbf{T}}\left(\mathbf{A}_{22} \mathbf{M}_{\mathbf{u}}^{-1} \mathbf{A}_{22}^{\mathbf{T}}\right)^{-1}\left(\mathbf{b}_{2}-\mathbf{A}_{22} \mathbf{a}_{2}-\mathbf{A}_{21} \mathbf{A}_{11}^{-1} \mathbf{b}_{1}\right) \\
& =\frac{1}{2} \mathbf{E}_{1} \mathbf{A}_{22}^{\mathbf{T}}\left(\mathbf{A}_{22} \mathbf{M}_{\mathbf{u}}^{-1} \mathbf{A}_{22}^{\mathbf{T}}\right)^{-1}\left(\mathbf{b}_{2}-\mathbf{A}_{22} \mathbf{a}_{2}-\mathbf{A}_{21} \mathbf{a}_{1}-\frac{1}{m} \mathbf{A}_{21} \mathbf{F}^{\mathbf{C}}\right)
\end{aligned}
$$

where Eq. (75) has been used in the last equality and Eq. (76) shows the relationship between the orbital control forces $\mathbf{F}^{\mathbf{C}}(\bar{t})$ and the attitude control torques $\Gamma^{\mathrm{C}}(t)$. If Eq. (66) is employed, the attitude control torque is alternatively given by 


$$
\begin{aligned}
& \boldsymbol{\Gamma}^{\mathbf{C}}(t)=2 \mathbf{E}_{1} \overline{\mathbf{A}}_{22}^{\mathbf{T}}\left(\overline{\mathbf{A}}_{22} \mathbf{E}_{1}^{\mathbf{T}} \mathbf{J}^{-1} \mathbf{E}_{1} \overline{\mathbf{A}}_{22}^{\mathbf{T}}\right)^{-1}\left(\overline{\mathbf{b}}_{2}-\overline{\mathbf{A}}_{22} \mathbf{a}_{2}-\overline{\mathbf{A}}_{21} \mathbf{A}_{11}^{-1} \mathbf{b}_{1}\right) \\
& =2 \mathbf{E}_{1} \overline{\mathbf{A}}_{22}^{\mathbf{T}}\left(\overline{\mathbf{A}}_{22} \mathbf{E}_{1}^{\mathbf{T}} \mathbf{J}^{-1} \mathbf{E}_{1} \overline{\mathbf{A}}_{22}^{\mathbf{T}}\right)^{-1}\left(\overline{\mathbf{b}}_{2}-\overline{\mathbf{A}}_{22} \mathbf{a}_{2}-\overline{\mathbf{A}}_{21} \mathbf{a}_{1}-\frac{1}{m} \overline{\mathbf{A}}_{21} \mathbf{F}^{\mathbf{C}}\right)
\end{aligned}
$$

From Eqs. (76) and (77), it is noted that the attitude control in general depends on the follower satellite's orbital tracking requirements.

Such a situation ( $\mathbf{A}_{11}$ is invertible) would occur, for example, if instead of Eq. (34a), the two constraint equations $y=\rho_{0} \cos \left(\omega_{0} t\right)$ and $z=\rho_{0} \sin \left(\overline{\omega_{0} t}\right)$, where $\omega_{0}$ is a given orbital frequency in the $y z$ plane of the Hill frame, are used to specify the orbital requirements for the $i$ th follower, so that along with Eq. (34b) there exist three independent orbital constraints (requirements). The invertibility of $\mathbf{A}_{11}$ assures that the three orbital requirements are independent of one another and the control force needed to satisfy them then becomes independent of the follower satellite's attitude. In brief, if there are three independent orbital constraints, then there is no need to consider any attitude constraints in designing the orbital controller.

Case 2: When $\mathbf{P}_{1} \mathbf{A}_{21}^{\mathbf{T}}=0$. First, it is noted that the third column of the $3 \times 3$ matrix $\mathbf{P}_{1} \mathbf{A}_{21}^{\mathbf{T}}$ is always zero because $\mathbf{A}_{21}^{\mathbf{T}}=\left[\begin{array}{ll}\overline{\mathbf{A}}_{21}^{\mathbf{T}} & 0_{3 \times 1}\end{array}\right]$. For the controller designed in the current paper, the orbital control is independent of the attitude control (or any attitude constraints) at each instant of time $t$ when there exists a $3 \times 3$ matrix $\mathbf{H}$ such that

$$
\mathbf{A}_{21}^{\mathbf{T}}=\mathbf{P}_{0} \mathbf{H}
$$

The following is the derivation of Eq. (78). The solution $\mathbf{A}_{21}^{\mathbf{T}}$ to the matrix equation $\mathbf{P}_{1} \mathbf{A}_{21}^{\mathbf{T}}=0$ is given by [39]

$$
\mathbf{A}_{21}^{\mathbf{T}}=\left(\mathbf{I}_{3 \times 3}-\mathbf{P}_{1}^{+} \mathbf{P}_{1}\right) \mathbf{H}
$$

where $\mathbf{H}$ is an arbitrary $3 \times 3$ matrix. With the fact that $\mathbf{P}_{1}^{+}=$ $\left(\mathbf{I}_{3 \times 3}-\mathbf{P}_{0}\right)^{+}=\left(\mathbf{I}_{3 \times 3}-\mathbf{A}_{11}^{+} \mathbf{A}_{11}\right)^{+}=\mathbf{I}_{3 \times 3}-\mathbf{A}_{11}^{+} \mathbf{A}_{11}=\mathbf{P}_{1}$ and the matrix $\mathbf{P}_{1}$ is idempotent, the right-hand side of Eq. (79) becomes $\mathbf{P}_{0} \mathbf{H}$, which proves Eq. (8). With Eq. (8), it follows that

$$
\begin{aligned}
\mathbf{P}_{1} \mathbf{A}_{21}^{\mathbf{T}} & =\mathbf{P}_{1} \mathbf{P}_{0} \mathbf{H}=\left(\mathbf{I}_{3 \times 3}-\mathbf{P}_{0}\right) \mathbf{P}_{0} \mathbf{H}=\left(\mathbf{P}_{0}-\mathbf{P}_{0}^{2}\right) \mathbf{H} \\
& =\left(\mathbf{P}_{0}-\mathbf{P}_{0}\right) \mathbf{H}=0
\end{aligned}
$$

so that the separation principle holds. This case is more limited than the previous one, in that Eq. (78) may be satisfied only at several time points during the maneuver. In Appendix A, it is shown that the $2 \times 3$ matrix $\overline{\mathbf{A}}_{21}$ always has full rank, that is, a rank of 2 . Then, the rank of the matrix $\mathbf{A}_{21}^{\mathbf{T}}$ is 2 and the rank of the matrix $\mathbf{P}_{0}$ is $s$, and so it is concluded that, from Eq. (그),

$$
\operatorname{rank}\left(\mathbf{A}_{21}^{\mathbf{T}}\right)=\operatorname{rank}\left(\mathbf{P}_{0} \mathbf{H}\right) \leq \operatorname{rank}\left(\mathbf{P}_{0}\right)
$$

which yields

$$
2 \leq s
$$

Equation (82) states that, for a matrix $\mathbf{H}$ to exist, the number of independent orbital constraints should be equal to or more than two. If $s=3$, the matrix $\mathbf{A}_{11}$ at the time $t$ is invertible, $\mathbf{P}_{1}=0$, and this falls under case 1 , and the separation principle always applies. If $s=2$, then $\operatorname{rank}\left(\mathbf{P}_{1}\right)=3-s=1$ from Eq. (61), so that the rank of the $3 \times 3$ matrix $\mathbf{P}_{1} \mathbf{A}_{21}^{\mathbf{T}}$ is zero or one. The rank of zero means that $\mathbf{P}_{1} \mathbf{A}_{21}^{\mathbf{T}}$ is a null matrix, Eq. (74) is satisfied, and the separation principle holds. The rank of one means that the first two nonzero columns of the $3 \times 3$ matrix $\mathbf{P}_{1} \mathbf{A}_{21}^{\mathbf{T}}$ are linearly dependent and the separation principle holds only at the time instants when there exists a three vector $\boldsymbol{\alpha}_{\mathbf{O}, \mathbf{A}}$, such that $\mathbf{P}_{1} \mathbf{A}_{21}^{\mathbf{T}} \boldsymbol{\alpha}_{\mathbf{O}, \mathbf{A}}=0$. Finally, if $s=1$, a

\begin{tabular}{|c|c|c|}
\hline$s$ & $\operatorname{Rank}\left(\mathbf{P}_{1} \mathbf{A}_{21}^{\mathbf{T}}\right)$ & Separation principle \\
\hline 1 & 1 & $\begin{array}{l}\text { Holds only at the time sections } \\
\text { when } \mathbf{P}_{1} \mathbf{A}_{21}^{\mathrm{T}} \alpha_{\mathbf{O}, \mathbf{A}}=0\end{array}$ \\
\hline & 2 & $\begin{array}{c}\text { Never holds as long as } \\
\qquad \boldsymbol{\alpha}_{\mathbf{O}, \mathbf{A}} \neq 0\end{array}$ \\
\hline 2 & $\begin{array}{l}0 \\
1\end{array}$ & $\begin{array}{l}\text { Holds } \\
\text { Holds only at the time points } \\
\text { when } \mathbf{P}_{1} \mathbf{A}_{21}^{\mathbf{T}} \boldsymbol{\alpha}_{\mathbf{O}, \mathbf{A}}=0\end{array}$ \\
\hline 3 & 0 & Always holds \\
\hline
\end{tabular}
matrix $\mathbf{H}$ does not exist and $\mathbf{P}_{1} \mathbf{A}_{21}^{\mathbf{T}}$ is never zero, and the rank of $\mathbf{P}_{1} \mathbf{A}_{21}^{\mathbf{T}}$ is one or two (because the third column of $\mathbf{P}_{1} \mathbf{A}_{21}^{\mathbf{T}}$ is always zero). When the rank is one, the separation principle holds only at the instants when $\mathbf{P}_{1} \mathbf{A}_{21}^{\mathbf{T}} \boldsymbol{\alpha}_{\mathbf{O}, \mathbf{A}}=0$ is satisfied as before. When the rank is two, $\mathbf{P}_{1} \mathbf{A}_{21}^{\mathbf{T}} \boldsymbol{\alpha}_{\mathbf{O}, \mathbf{A}} \neq 0$ as long as $\boldsymbol{\alpha}_{\mathbf{O}, \mathbf{A}} \neq 0$ because no linear
Table 1 Various conditions for the separation principle depending on the rank $s$ of $\mathrm{A}_{11}$

combination of the two nonzero columns of $\mathbf{P}_{1} \mathbf{A}_{21}^{\mathbf{T}}$ makes $\mathbf{P}_{1} \mathbf{A}_{21}^{\mathbf{T}} \boldsymbol{\alpha}_{\mathbf{O}, \mathbf{A}}$ zero, which means that the separation principle never holds. The results are summarized in Table 1 .

Remark: One of the strengths of the analytical solution is that it is possible to estimate in advance the magnitude or the order of the required control force and/or torque. For example, the second component of $\mathbf{F}^{\mathbf{C}}(t)$ is

$$
m \mathbf{P}_{1} \overline{\mathbf{A}}_{21}^{\mathbf{T}} \overline{\boldsymbol{\alpha}}_{\mathbf{O}, \mathbf{A}}=m \mathbf{P}_{1} \overline{\mathbf{A}}_{21}^{\mathbf{T}} \overline{\mathbf{D}}\left(\overline{\mathbf{b}}_{2}-\overline{\mathbf{A}}_{21} \mathbf{a}_{1}-\overline{\mathbf{A}}_{22} \mathbf{a}_{2}-\overline{\mathbf{A}}_{21} \boldsymbol{\alpha}_{\mathbf{O}}\right)
$$

from Eq. (드). With the definitions of each term in the right-hand side of Eq. (83), the matrix norm of $\overline{\mathbf{D}}$ is very small if the absolute distance between the follower satellite and the target, that is, $\|\boldsymbol{\xi}(t)\|=$ $\left\|\mathbf{X}_{\mathbf{t}}(t)-\mathbf{X}(t)\right\|$ is sufficiently large. For example, provided that the target is set as the center of the Earth, $\|\boldsymbol{\xi}(t)\|$ is of the order of $10^{6}$ (in meters) if the follower satellite is in low Earth orbit. Then, the matrix norm of $\overline{\mathbf{A}}_{22}$, which is almost the same as $\|\boldsymbol{\xi}(t)\|$, is also large, which makes the norm of $\overline{\mathbf{D}}$ very small because $\overline{\mathbf{D}}$ is the inverse of the matrix multiplication of two $\overline{\mathbf{A}}_{22}$ matrices. The norms of the elements in the parentheses (i.e., $\left\|\overline{\mathbf{b}}_{2}\right\|,\left\|\overline{\mathbf{A}}_{21} \mathbf{a}_{1}\right\|,\left\|\overline{\mathbf{A}}_{22} \mathbf{a}_{2}\right\|$, and $\left\|\overline{\mathbf{A}}_{21} \boldsymbol{\alpha}_{\mathbf{O}}\right\|$ ) of the right-hand side of Eq. ( $\underline{83})$ are much smaller than $\|\boldsymbol{\xi}(t)\|$ because the norm of $\mathbf{a}_{2}$ is of the order of the norm of $\|\dot{\mathbf{u}}\|^{2}$, and the norms $\left\|\mathbf{P}_{1}\right\|$ and $\left\|\overline{\mathbf{A}}_{21}\right\|$ are both of the order unity. It is therefore concluded that $m \mathbf{P}_{1} \overline{\mathbf{A}}_{21}^{\mathbf{T}} \overline{\boldsymbol{\alpha}}_{\mathbf{O}, \mathbf{A}}$ is a lot smaller than $m \alpha_{\mathbf{O}}$. Hence, in many cases, the first component $m \alpha_{\mathbf{O}}$ dominates the second component $m \mathbf{P}_{1} \overline{\mathbf{A}}_{21}^{\mathbf{T}} \overline{\boldsymbol{\alpha}}_{\mathbf{O}, \mathbf{A}}$ in the control force $\mathbf{F}^{\mathbf{C}}(t)$ for orbital control.

For reference, the attitude control torques are described by $\boldsymbol{\Gamma}^{\mathbf{C}}(t)=(m / 2) \mathbf{E}_{1} \overline{\mathbf{A}}_{22}^{\mathbf{T}} \overline{\boldsymbol{\alpha}}_{\mathbf{O}, \mathbf{A}}$ from Eq. (66) and it is shown in Appendix $\underline{A}$ that the rank of the matrix $\overline{\mathbf{A}}_{22} \mathbf{E}_{1}^{\mathbf{T}}$ has full rank (i.e., rank of two), so that $\boldsymbol{\Gamma}^{\mathbf{C}}(t)$ is never zero as long as $\overline{\boldsymbol{\alpha}}_{\mathbf{O}, \mathbf{A}}$ is not zero. Also, although $\overline{\boldsymbol{\alpha}}_{\mathbf{O}, \mathbf{A}}=\overline{\mathbf{D}}\left(\overline{\mathbf{b}}_{2}-\overline{\mathbf{A}}_{21} \mathbf{a}_{1}-\overline{\mathbf{A}}_{22} \mathbf{a}_{2}-\overline{\mathbf{A}}_{21} \boldsymbol{\alpha}_{\mathbf{O}}\right)$ may be small as stated earlier, the torque $\boldsymbol{\Gamma}^{\mathbf{C}}(t)$ is not negligible because the matrix norm of $\overline{\mathbf{A}}_{22}$ is large (of the order of $\|\boldsymbol{\xi}\|$ ).

\section{Numerical Example}

In this section, an example is introduced to demonstrate the applicability of the control methodology suggested in the preceding sections. It is straightforward to extend this example for applications to more general situations. The numerical integration throughout this paper is done in the MATLAB environment, using a variable time step $(4,5)$-modified Runge-Kutta integrator with a relative error tolerance of $10^{-13}$ and an absolute error tolerance of $10^{-16}$.

Let us consider a formation system in which there is one follower satellite whose mass is $m=6.5 \mathrm{~kg}$. Also, its moments of inertia along its respective body-fixed axes are taken to be $\mathbf{J}=$ $\operatorname{diag}\left(\begin{array}{lll}1.88 & 1.88 & 1.1\end{array}\right) \mathrm{kg} \cdot \mathrm{m}^{2}$. It is assumed that the leader satellite is in a $J_{2}$-perturbed circular reference orbit around the oblate Earth whose initial radius is $r_{L}=7.1781363 \times 10^{6} \mathrm{~m}$ (i.e., the altitude is $800 \mathrm{~km}$ ). The initial right ascension of the ascending node $\Omega_{L}$ and the inclination $i_{L}$ of the leader satellite's orbit are 30 and $80 \mathrm{deg}$, respectively, and it is assumed that the leader satellite is at the ascending node at the initial time $(t=0)$. The leader satellite's "nominal" orbital frequency and its nominal orbital period (the orbit is not closed) are given by, respectively, 


$$
\begin{aligned}
& n_{L}=\sqrt{\frac{G M_{\oplus}}{r_{L}^{3}}}=1.0381292 \times 10^{-3} \mathrm{rad} / \mathrm{s}, \\
& P_{L}=\frac{2 \pi}{n_{L}}=6.0524127 \times 10^{3} \mathrm{~s}=1.6812257 \mathrm{~h}
\end{aligned}
$$

and $2 P_{L}$ is chosen as the duration of time used for numerical integration. Figure 1 shows the leader satellite's trajectory in the ECI frame. The trajectory in three-dimensional space is given in the upper left figure. The upper right, the lower left, and the lower right figures show the trajectories projected onto the $X Y, Y Z$, and $X Z$ planes, respectively. The trajectory is not closed due to the $J_{2}$ perturbation, although it is difficult to see this from the figure.

For orbital control, the follower satellite, which is also under the $J_{2}$ perturbation, is required to satisfy Eq. (34) or stay on a circle with a constant radius $\rho_{0}=2 \mathrm{~km}$ when projected onto the $y z$ plane in the Hill frame (centered at the leader satellite). As an attitude requirement, the $z$ axis of the body frame of the follower satellite is required to point to the center of the leader satellite at all times, that is, $\mathbf{X}_{\mathbf{t}}(t)$ is the position vector of the leader satellite. One of the correct initial conditions for orbital motion of the follower satellite in the Hill frame is given by

$$
\begin{aligned}
& x(0)=0 \mathrm{~m}, \quad y(0)=2000 \mathrm{~m}, \\
& z(0)=0 \mathrm{~m} \quad \dot{x}(0)=1.0381290 \mathrm{~m} / \mathrm{s}, \\
& \dot{y}(0)=-0.02076223 \mathrm{~m} / \mathrm{s}, \quad \dot{z}(0)=2.0762581 \mathrm{~m} / \mathrm{s}
\end{aligned}
$$

However, because the relative initial position and velocity should be measured, for example, from a carrier-phase differential GPS sensor, they may be corrupted by sensor noise. With filtered carrier-phase differential GPS signals, the relative position measurements are predicted to be accurate to approximately $2-5 \mathrm{~cm}$ and the velocity noise is predicted to be on the order of $2-3 \mathrm{~mm} / \mathrm{s}$ [40]. In the current paper, for illustration purposes, let us consider measurement errors in excess of these estimates. The errors in the initial position are taken to be $+\sqrt{6} \mathrm{~cm}$ in the radial direction ( $x$ direction in the Hill frame) and $+\sqrt{6} \mathrm{~cm}$ in the in-track direction ( $y$ direction in the Hill frame), and the errors in the initial velocity are taken to be +2 and $+2 \mathrm{~mm} / \mathrm{s}$, respectively, in the radial and in-track directions, that is,

$$
\begin{aligned}
& x(0)=0.0244949, \quad y(0)=2000.0244949, \\
& z(0)=0 \quad \dot{x}(0)=1.0401290, \quad \dot{y}(0)=0.002, \\
& \dot{z}(0)=2.0762581
\end{aligned}
$$
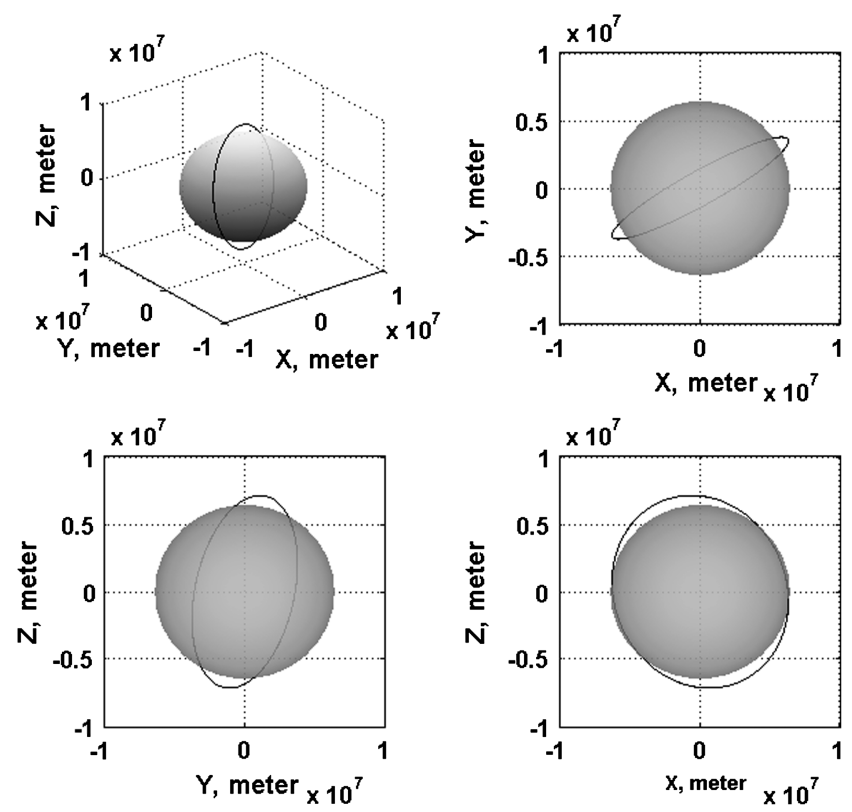

Fig. 1 Leader satellite's trajectory in the ECI frame. and Eq. (6) can be transformed into the ones in the ECI frame using Eq. (16):

$$
\begin{array}{lrl}
X(0) & =6.2162748 \times 10^{6}, & Y(0)=3.5893689 \times 10^{6}, \\
Z(0) & =1.9696396 \times 10^{3} & \dot{X}(0)=-646.8736516, \\
\dot{Y}(0) & =1118.3457218, & \dot{Z}(0)=7338.9841623
\end{array}
$$

The initial conditions for attitude motion are chosen as

$$
\begin{aligned}
& u_{0}(0)=0.07073720, \quad u_{1}(0)=0.9915440, \\
& u_{2}(0)=0.1024636, \\
& u_{3}(0)=0.0365786 \quad \dot{u}_{0}(0)=-5.6671227 \times 10^{-4}, \\
& \dot{u}_{1}(0)=2.8402648 \times 10^{-6}, \quad \dot{u}_{2}(0)=3.6375241 \times 10^{-4}, \\
& \dot{u}_{3}(0)=0
\end{aligned}
$$

These initial conditions do not satisfy the attitude constraints $(45 \mathrm{c})$ and (45d), but do satisfy the unit-norm constraint (47) to describe a physical rotational motion. Thus, sensor errors in attitude measurements are also included. More specifically, if $\theta$ is defined as the angle between the vector $\hat{\mathbf{z}}_{\text {body }}$ and the vector $\mathbf{T} \boldsymbol{\xi}$ connecting the follower satellite and the target (the leader satellite), that is, $\theta:=$ $\angle\left(\hat{\mathbf{z}}_{\text {body }}, \mathbf{T} \boldsymbol{\xi}\right.$ ) (see Fig. A1 in Appendix A), then $\theta$ is approximately $\theta(0) \simeq 1.02 \mathrm{deg}$ at the initial time; exact satisfaction of the attitude constraints would require $\theta$ to be zero [see Eq. (45)]. It should be noted that, because sensor measurement noise is present, affecting the correct values of the initial conditions, both in orbit and attitude, Eq. (63) should be used to obtain the control force and torque [instead of Eq. (69)] and Eq. (70) should be used to obtain the controlled position and velocity history of the follower satellite [instead of Eq. (71)]. The parameter values, $\alpha_{i}=0.002$ and $\beta_{i}=0.002(i=1$, $2,3,4,5)$ are chosen in the constraint equations (8).

Figure 2 represents the orbits of the follower satellite with control projected on the $y z$ and $x z$ planes in the Hill frame, respectively. The scale is normalized by $\rho_{0}$ and these trajectories are obtained by integrating twice the analytical results given in Eq. (70). Although it is not obvious from this figure, there are small initial discrepancies from the desired formation configuration of the follower satellite due to the incorrect initial conditions caused by sensor measurement errors. Despite these measurement errors, asymptotic convergence to the desired orbital and attitude trajectories occurs, which will be discussed later. As seen in Fig. 2, the follower satellite quickly moves on the constraint circle of (normalized) radius unity and satisfies the linear constraint as required by Eq. (34). Figure 3 depicts the orbits of the follower satellite without control projected on the $y z$ and $x z$ planes in the Hill frame, respectively. As seen from the figures, without control, the follower satellite appears to show unbounded motion and moves leftward in the $y$ direction, necessitating control.

In Fig. 4, the time histories of each component of the quaternion for the follower satellite with control are shown where time is normalized by $P_{L}$, the nominal period of the leader satellite [see Eq. (84)]. Figure $\underline{5}$ depicts quaternion time histories for the follower satellite without control. As expected, each component starts with its initial quaternion value and with the initial slope of its quaternion derivative, and each maintains this oscillation in the absence of any control force and/or torque.

Figure 6 depicts the control forces $\mathbf{F}^{\mathbf{C}}(t)$ per unit mass of the follower satellite to maintain the desired orbital formation. These forces are directly obtained using the analytical results, Eq. (63). The force components are described in the ECI frame, and time is normalized by the nominal period of the leader satellite (i.e., $P_{L}$ ). As seen, relatively large control forces are brought into play initially to eliminate the initial errors in the orbit of the follower satellite. After this initial adjustment, as seen from the figure, the forces required for orbital control become small. One can reduce the magnitude of the control force by choosing smaller values of $\alpha_{i}$ and $\beta_{i}$ than those used here; but this would, of course, come at the expense of a longer time 

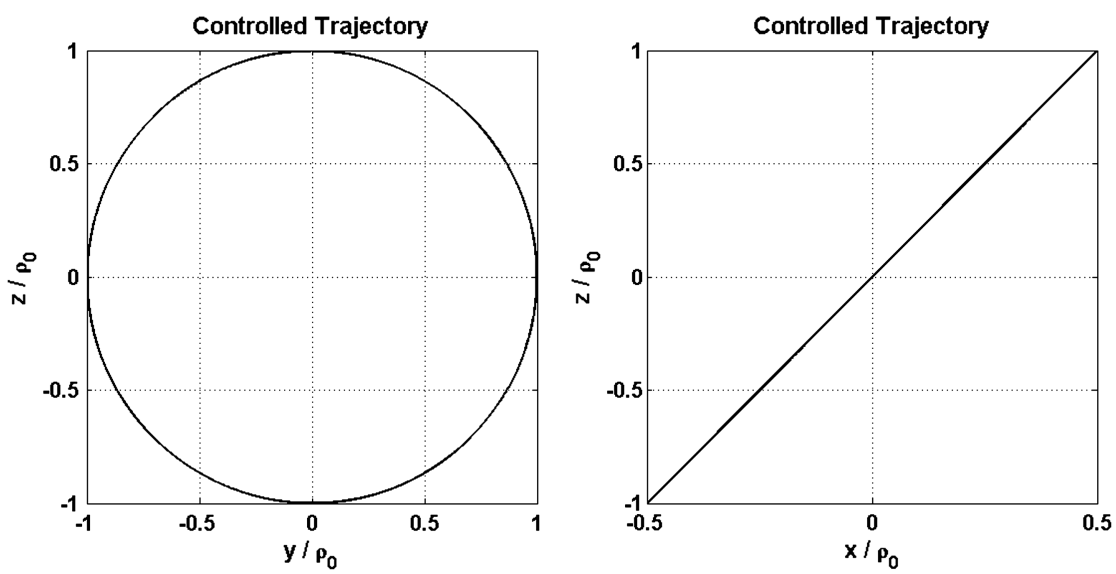

Fig. 2 Controlled orbital motion in the $y z$ and $x z$-planes of the follower satellite.
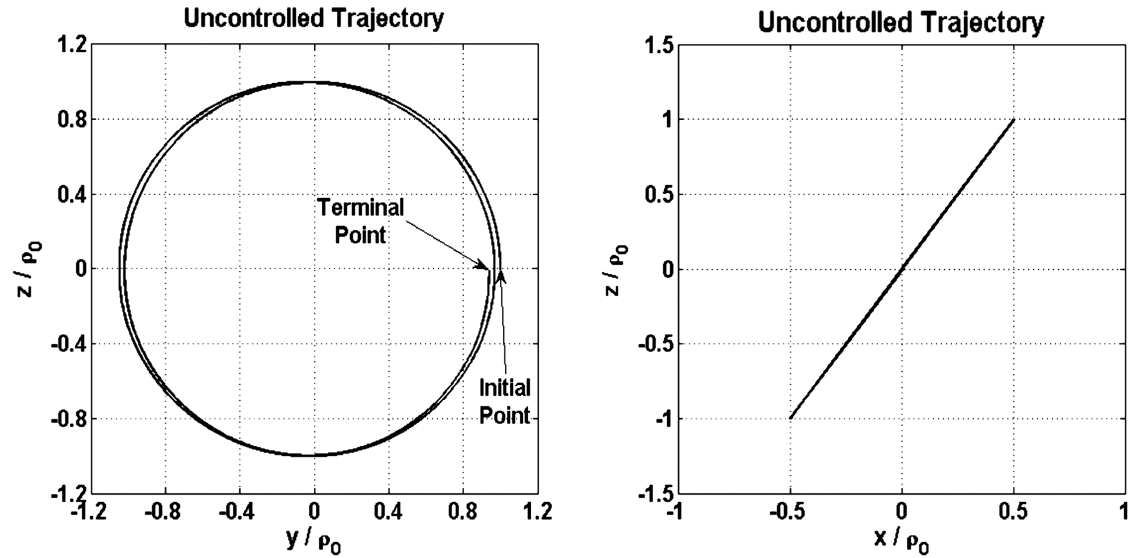

Fig. 3 Uncontrolled orbital motion in the $y z$ and $x z$-planes of the follower satellite.

duration needed to get the follower satellite to its required trajectory. Figure 7 illustrates the control torques $\Gamma^{\mathrm{C}}(t)$ per unit mass of the follower satellite for satisfying the attitude requirements. These torques are also directly obtained using the new analytical results, Eq. (63). The torque components are described in the body frame of the follower satellite, and it is seen again that relatively large control torques are required to mitigate initial attitude errors. From the figure, little torque along the $z$ axis is needed once the target pointing becomes accurate. This means that only the $x$ and $y$ components are necessary to control the orientation of the follower satellite. This result can be derived from the analytical solution given in Eq. (66). From Eq. (A4) of Appendix A, it is seen that the third row of the matrix $\mathbf{E}_{1} \overline{\mathbf{A}_{22}^{\mathbf{T}}}$ is zero because $\mathbf{T}_{1} \boldsymbol{\xi}=\mathbf{T}_{2} \boldsymbol{\xi}=0$ if the attitude constraints are satisfied by Eqs. $(\underline{45 \mathrm{c}})$ and $(\underline{45 \mathrm{~d}})$, and so the $z$ component of the control torque is identically zero.

Figure 8 represents errors in the satisfaction of the desired trajectory requirements in the presence of sensor measurement errors. The first error plotted is $e_{1}(t):=\sqrt{\left(\mathbf{R}_{2} \mathbf{X}\right)^{2}+\left(\mathbf{R}_{3} \mathbf{X}\right)^{2}}-\rho_{0}$ [see Eq. (35a)] and the second error $e_{2}(t):=2\left(\mathbf{R}_{1} \mathbf{X}-r_{L}\right)-\mathbf{R}_{3} \mathbf{X}$ [see Eq. $(\underline{35 b})]$, both being measured in meters. The third error is the

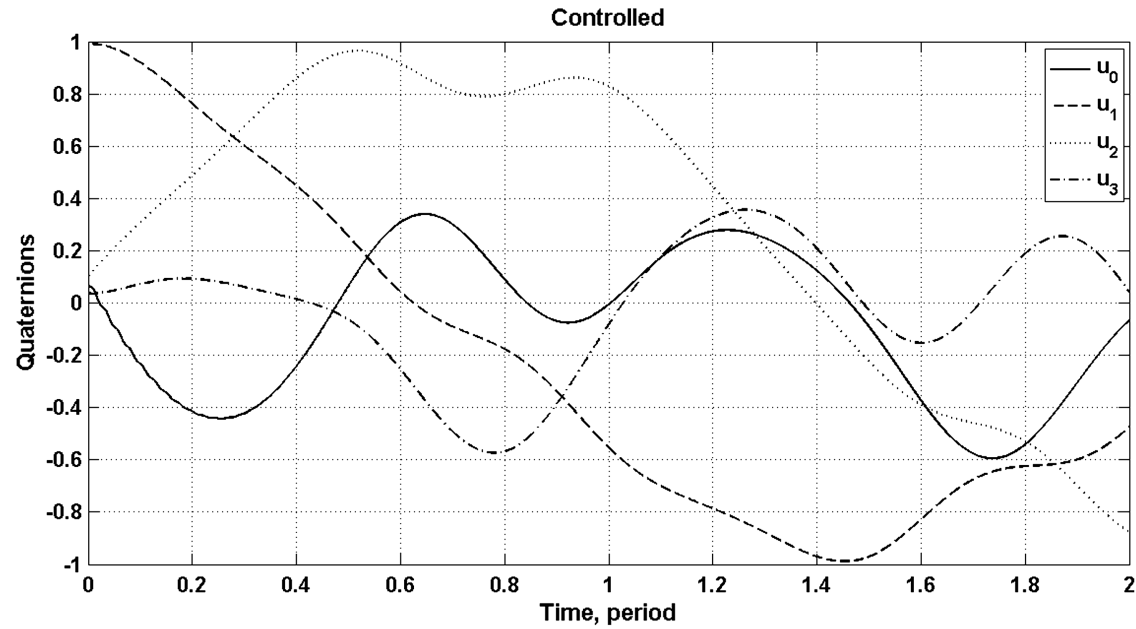

Fig. 4 Quaternion of the follower satellite with control. 


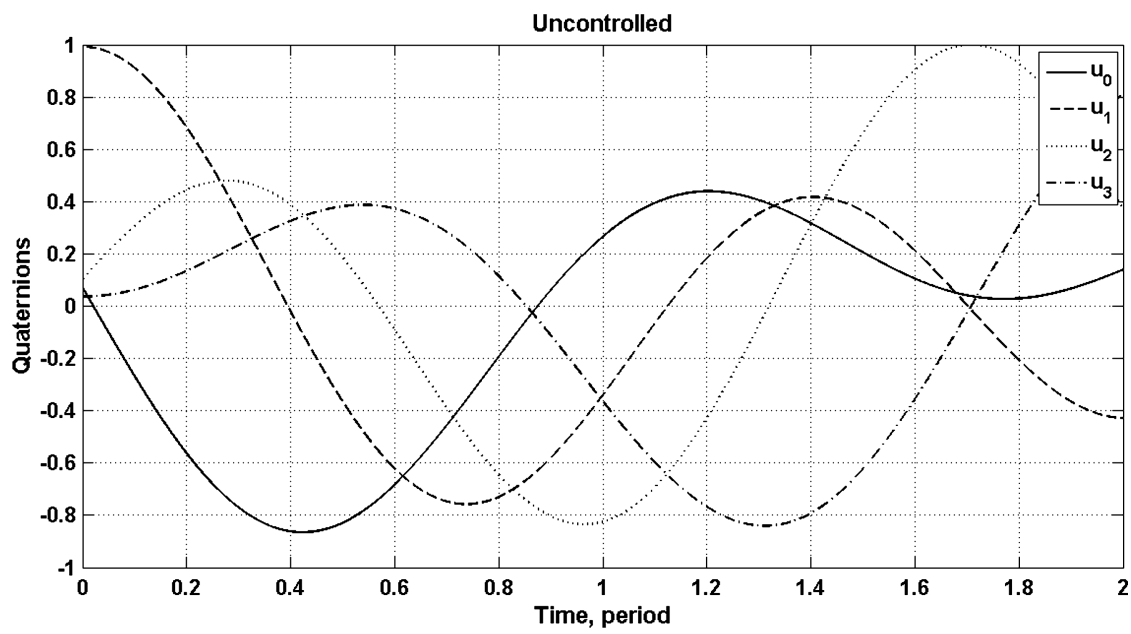

Fig. 5 Quaternion of the follower satellite without control.
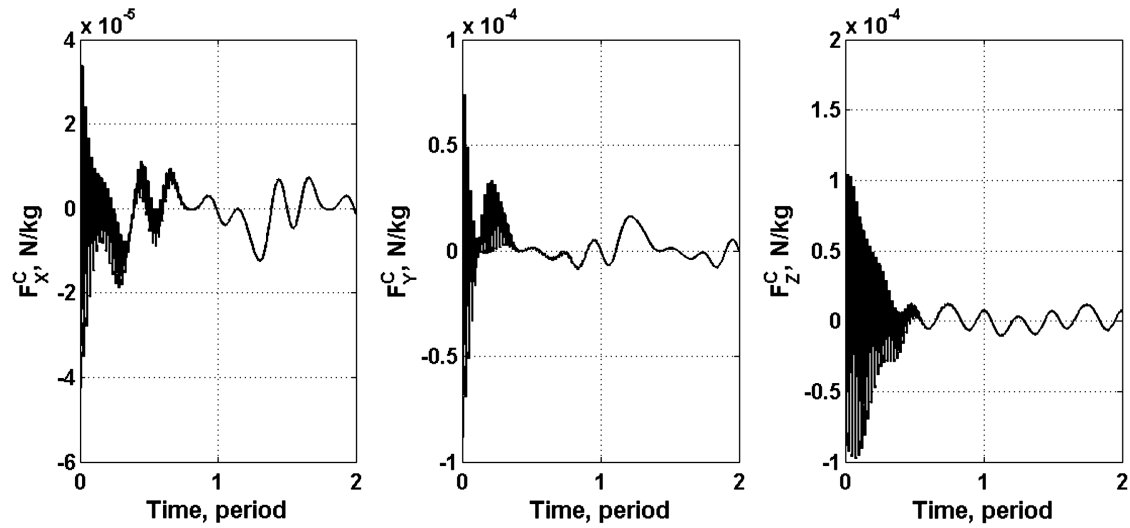

Fig. 6 Required control forces per unit mass in the ECI frame for the follower satellite from Eq. (63).
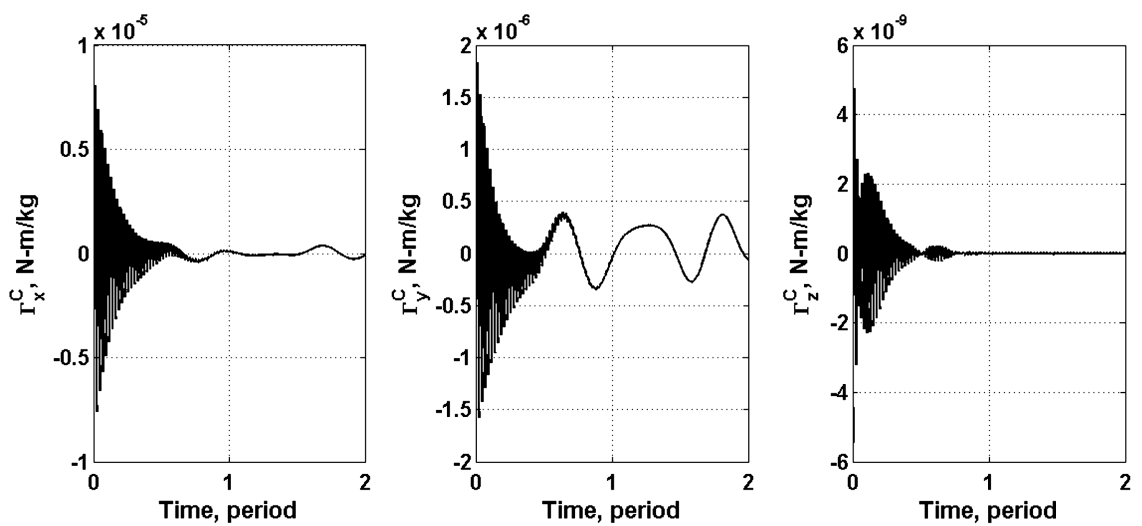

Fig. 7 Required control torques per unit mass in the body frame for the follower satellite from Eq. (63).
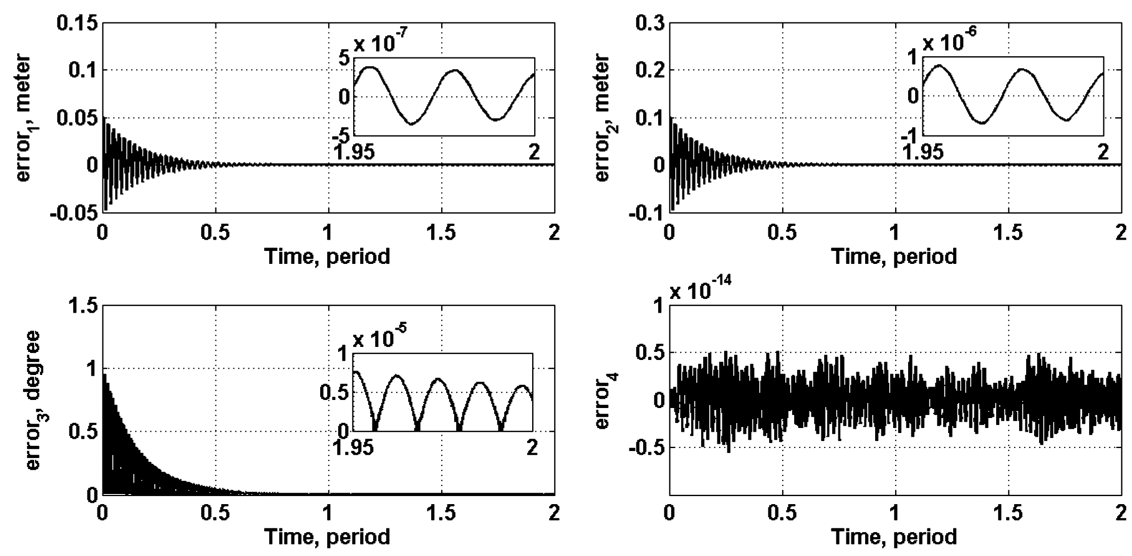

Fig. 8 Errors in orbital and attitude requirements with control and sensor measurement errors. 
pointing error. As previously defined, $\theta$ is the angle between the vector $\hat{\mathbf{z}}_{\text {body }}$ and the vector $\mathbf{T} \boldsymbol{\xi}$, so that $e_{3}(t):=\theta$ in degrees, which is required to be zero. The fourth error is the unit-norm quaternion constraint $e_{4}(t)=u_{0}^{2}+u_{1}^{2}+u_{2}^{2}+u_{3}^{2}-1$. Initially, the first three errors are large because the incorrect initial conditions, because of sensor measurement noise, are used; however, they asymptotically converge to zero, as expected, as time progresses. The small boxes that magnify the errors over the duration $1.95 P_{L}-2 P_{L}$ confirm this; the final errors in the satisfaction of the desired trajectory requirements are of the order of $10^{-7}, 10^{-7}, 10^{-6}$, and $10^{-15}$, respectively. Even in the presence of the incorrect initial conditions, the final orbital tracking errors are remarkably small, when compared with existing results. In Ref. [32] in which linear programming is employed, the authors require the follower satellite to stay around the leader satellite within an error box with dimensions of several meters. Also, for comparison, the same errors are plotted in Fig. 9, in which no control forces and no control torques are applied. As expected, it is seen that the errors are very large by several orders of magnitude.

The analytical results obtained in Sec. IV related to the separation principle can provide new insights into the control dynamics. Because there are two independent orbital constraints (34a) and (34b) in this example (i.e., $s=2$ ), from Table 1 the rank of $\mathbf{P}_{1} \overline{\mathbf{A}_{21}^{\mathbf{T}}}$ should be zero or one during the maneuver. When the rank is zero, the separation principle holds and when the rank is one, the two nonzero column vectors of $\mathbf{P}_{1} \mathbf{A}_{21}^{\mathbf{T}}$ are linearly dependent and the separation principle holds only at the instants when $\mathbf{P}_{1} \mathbf{A}_{21}^{\mathbf{T}} \boldsymbol{\alpha}_{\mathbf{O}, \mathbf{A}}=0$ is satisfied. According to the numerical computation, the rank of $\mathbf{P}_{1} \mathbf{A}_{21}^{\mathbf{T}}$ is always one throughout the maneuver in this example. Fig. 10 shows that the separation principle appears to apply only at isolated times, as happens for the first time at $t=0.0058367 P_{L}$, at which time the three components of the vector $\mathbf{P}_{1} \mathbf{A}_{21}^{\mathbf{T}} \alpha_{\mathbf{O}, \mathbf{A}}$ [the second term on the righthand side in Eq. (55)] simultaneously become zero. At the beginning, the separation principle applies relatively frequently (see the upper inset box in Fig. 10), but as the errors become small, the frequency with which it applies decreases (see the lower inset box). At times, other than when $\mathbf{P}_{1} \mathbf{A}_{21}^{\mathbf{T}} \boldsymbol{\alpha}_{\mathbf{O}, \mathbf{A}}=0$ the orbital control is not, strictly speaking, independent of the attitude of the follower satellite, though the contribution of $\mathbf{P}_{1} \mathbf{A}_{21}^{\mathbf{T}} \boldsymbol{\alpha}_{\mathbf{O}, \mathbf{A}}$ is several orders of magnitude smaller than that of the first term $\alpha_{\mathbf{O}}$ [see Eq. (63)]. Comparison with Fig. $\underline{6}$ shows that the first term $\boldsymbol{\alpha}_{\mathbf{O}}$, which is of the order of $10^{-4}$, mostly determines the orbital control force $\mathbf{F}^{\mathbf{C}}(t)$.

As a final application of the approach developed in this paper, the question of robustness under unmodeled dynamics is addressed. Though a full treatment of this topic would take us far afield, here only "small" unmodeled dynamics is considered and a simple approach is presented for robust control. The analytical results obtained herein can then be thought of as reference inputs in the presence of small external disturbances. In the presence of disturbances, the displacement vector $\mathbf{q}(t)$ of the follower satellite will differ from its reference displacement $\mathbf{q}_{\text {ref }}(t)$, which is obtained by integrating Eq. (70) twice, assuming no disturbances. If the error $\mathbf{e}(t):=\mathbf{q}_{\text {ref }}(t)-\overline{\mathbf{q}}(t)$ is small, then linear control theory would apply, and the closed-form analytically obtained control force [obtained from Eq. (63)] can be simply augmented by a PD controller, so that

$$
\mathbf{M} \ddot{\mathbf{q}}=\mathbf{Q}+\mathbf{Q}^{\mathbf{C}}+\left(\tilde{\mathbf{Q}}+K_{D} \dot{\mathbf{e}}+K_{P} \mathbf{e}\right)
$$
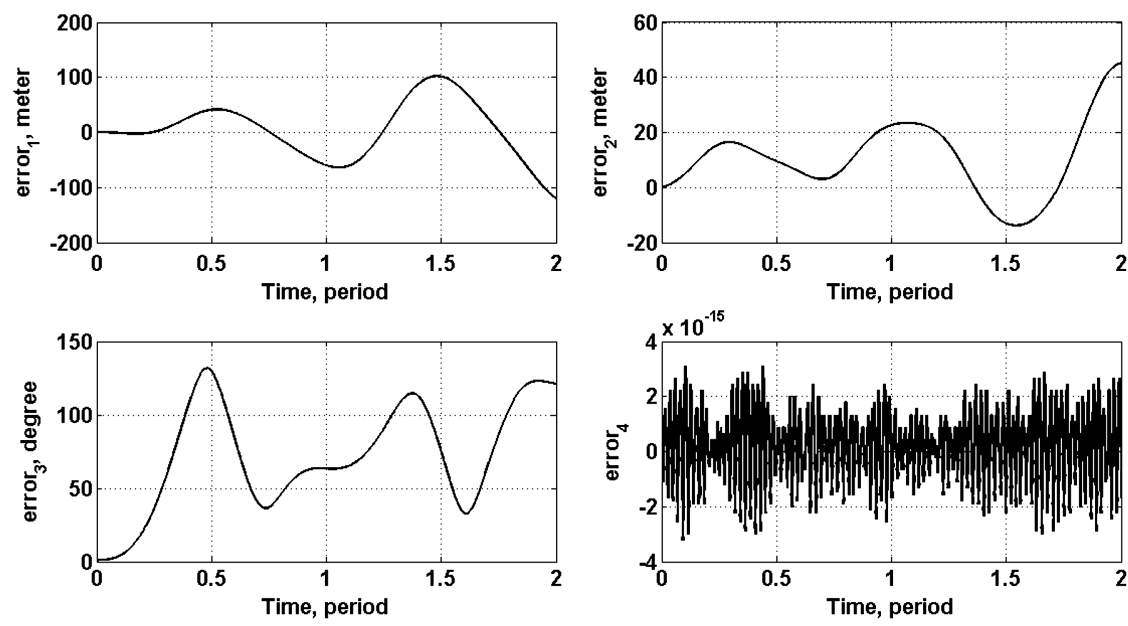

Fig. 9 Errors in orbital and attitude requirements with no control.

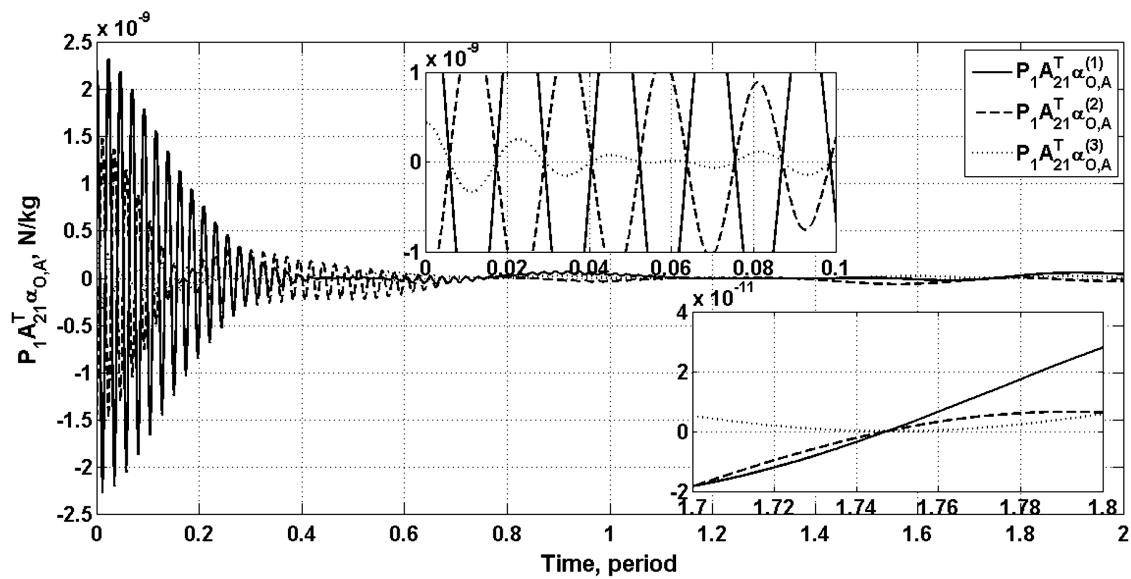

Fig. 10 Each component of the $P_{1} A_{21}^{T} \alpha_{O, A}$ to check if the separation principle holds. 


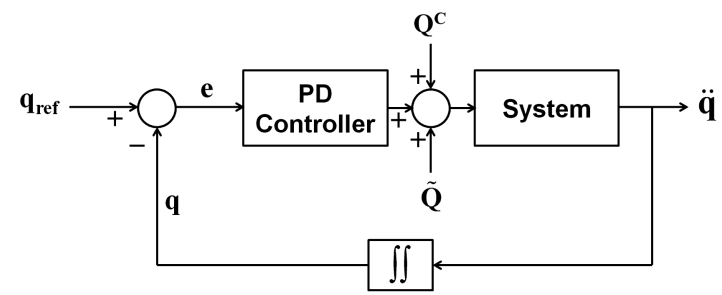

Fig. 11 Block diagram showing compensation for unmodeled dynamics using a PD controller for robust control.

where $\mathbf{M}$ is the mass matrix, $\mathbf{q}$ is the generalized displacement vector required to track $\mathbf{q}_{\text {ref }}, \mathbf{Q}$ is the given (impressed) force, $\mathbf{Q}^{\mathbf{C}}$ is the nominal control force and torque analytically obtained by Eq. (63), and $\tilde{\mathbf{Q}}$ is the unmodeled external disturbance. The parameters $K_{D}$ and $K_{P}$ are the control gains of the PD controller. Figure 11 illustrates a block diagram showing the error correction that is embedded in Eq. (89). For example, assume that the following orbital disturbance force $\mathbf{Q}$ applies to the follower satellite:

$\tilde{\mathbf{Q}}=\left[\begin{array}{lllllll}f_{D}+0.1 f_{D} \sin \left(n_{L} t\right) & f_{D}+0.1 f_{D} \cos \left(n_{L} t\right) & f_{D} & 0 & 0 & 0 & 0\end{array}\right]^{\mathbf{T}}$

where $f_{D}=2.418 \times 10^{-6} \mathrm{~N}$ and $n_{L}$ is the nominal orbital frequency of the leader satellite given in Eq. (84). Figure 12 shows the results

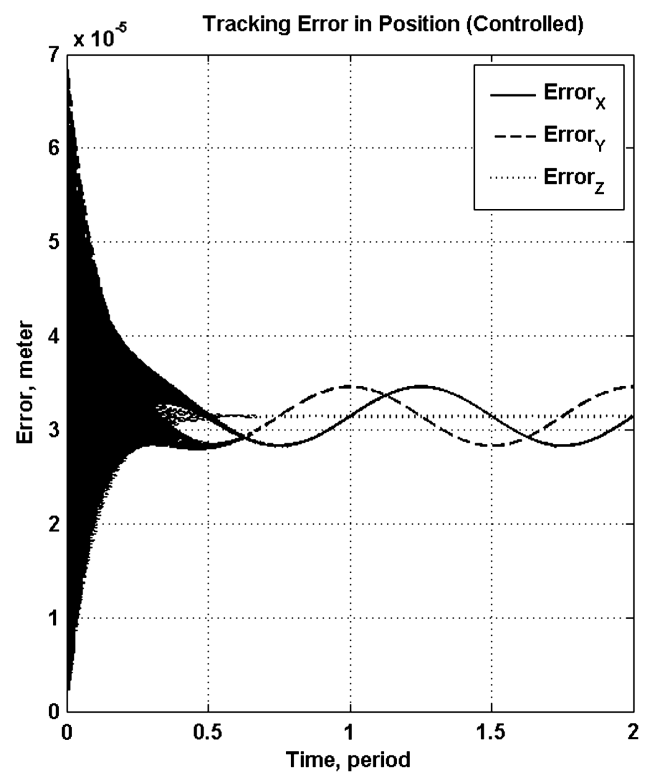

Fig. 12 Tracking errors in position showing robust control in the presence of small unmodeled dynamics. Note the vertical scale. when using an add-on PD controller [Eq. (89)] with gains of $K_{D} \overline{\tilde{\mathbf{Q}}}$ $0.02 \mathrm{~kg} / \mathrm{s}$ and $K_{P}=0.5 \mathrm{~kg} / \mathrm{s}^{2}$ to compensate for the disturbance $\tilde{\mathbf{Q}}$ given by Eq. (90). As seen, each component of the position vector is bounded with steady-state tracking errors of the order of $10^{-5} \mathrm{~m}$. Figure 13 shows the control forces per unit mass exerted by the PD controller. The magnitude of each component is much smaller than that shown in Fig. 6 , and hence adding this PD controller to the original controller designed using Eq. (63) would lead to robust control provided, of course, that the control design requirements permit the presence of such small steady-state tracking errors.

\section{Conclusions}

In this paper, a simple analytical solution for the precision formation-keeping problem, which also considers attitude requirements, is presented. The leader satellite is assumed to be in a $J_{2}$ perturbed circular reference orbit, and each of the $N$ follower satellites, which are also under the $J_{2}$ perturbation, is required to track different orbital and time-varying attitude requirements. The method fully preserves the complete nonlinearities of the dynamic system and handles both orbital and attitude dynamics in a simple and unified way. Even in the presence of sensor measurement noise, which causes errors in the initial conditions pertaining to both orbital and attitudinal motion, the methodology developed here provides a nonlinear closed-form controller that asymptotically tracks both the orbital and attitude mission requirements with very high accuracy. Also, because the orbital constraints and the attitude target pointing constraints are independent of each other, as shown in Appendix A, it is possible to obtain the explicit form of the solutions, which enables further insights into the dynamics of a multiple-satellite system. Specifically, it is shown under what conditions the attitude dynamics can be ignored, or separated away, when designing a controller for exact orbital control, leading to the statement of the separation principle. Some cases wherein the separation principle holds are summarized in Table 1 . As a special case, if there are three independent orbital constraints given, the separation principle is always satisfied throughout the maneuver. Two types of solutions, Eqs. (63) and (69), are presented depending on whether the attitude constraints and the unit-norm constraint [Eqs. (45c), (45d), and (47)] are satisfied; this would depend on the presence of attitude sensor measurement errors. The simpler solution, Eq. (69), results from the absence of attitude sensor measurement errors when the attitude constraints are exactly satisfied. In addition, the method is easy to computationally implement and yields results with high numerical accuracy. A numerical example that includes sensor measurement errors shows the simplicity, efficacy, and robustness of the approach. Via this example, it is also shown that the analytical results related to the closed-form controller obtained in this paper can be easily used for robust control in the presence of unmodeled dynamics by adding to this closed-form controller an additional simple PD controller to mitigate the effect of small unknown disturbances.
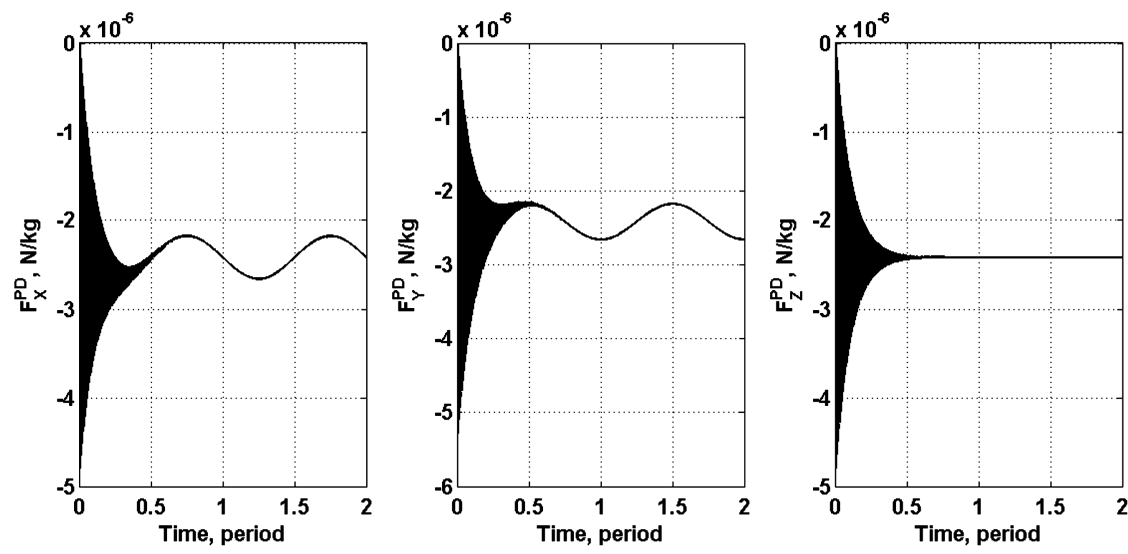

Fig. 13 Control forces per unit mass exerted by the PD controller. 


\section{Appendix A: Rank of Matrix A}

Now it is shown that the matrix A in Eq. (53) always has full rank, as long as $\mathbf{A}_{11}$ is full rank. The assumption that $\mathbf{A}_{11}$ has full rank is equivalent to assuming that the orbital tracking requirements for the $i$ th follower satellite are independent of one another.

Let us first show that the matrix $\overline{\mathbf{A}}_{21}$ always has full rank, that is, a rank of two. From the definition of $\hat{\mathbf{A}}_{21}$ in Eq. (50),

$$
\overline{\mathbf{A}}_{21}=\left[\begin{array}{c}
\mathbf{T}_{2} \\
-\mathbf{T}_{1}
\end{array}\right]
$$

Because the transformation matrix

$$
\mathbf{T}=\left[\begin{array}{l}
\mathbf{T}_{1} \\
\mathbf{T}_{2} \\
\mathbf{T}_{3}
\end{array}\right]
$$

is orthogonal, it is obvious that the two rows of $\overline{\mathbf{A}}_{21}$ are linearly independent, which proves that $\overline{\mathbf{A}}_{21}$ has full rank.

Next, it is shown that the $3 \times 4$ matrix $\mathbf{A}_{22}$ always has full rank, namely a rank of three. Because the matrix $\mathbf{E}$ [see Eq. (21)] is orthogonal, $\operatorname{rank}\left(\mathbf{A}_{22}\right)=\operatorname{rank}\left(\mathbf{A}_{22} \mathbf{E}^{\mathbf{T}}\right)$, and from Eqs. (21) and $(\underline{51})$, one has

$$
\mathbf{A}_{22} \mathbf{E}^{\mathbf{T}}=\left[\begin{array}{c}
\overline{\mathbf{A}}_{22} \\
2 \mathbf{u}^{\mathbf{T}}
\end{array}\right]\left[\begin{array}{ll}
\mathbf{u} & \mathbf{E}_{1}^{\mathbf{T}}
\end{array}\right]=\left[\begin{array}{cc}
\overline{\mathbf{A}}_{22} \mathbf{u} & \overline{\mathbf{A}}_{22} \mathbf{E}_{1}^{\mathbf{T}} \\
2 & 0_{1 \times 3}
\end{array}\right]
$$

because $\mathbf{u}^{\mathbf{T}} \mathbf{u}=1$ (assuming that the unit-norm constraint is satisfied throughout the maneuver to realize physical rotations) and $\mathbf{E}_{1} \mathbf{u}=0_{3 \times 1}$. Also,

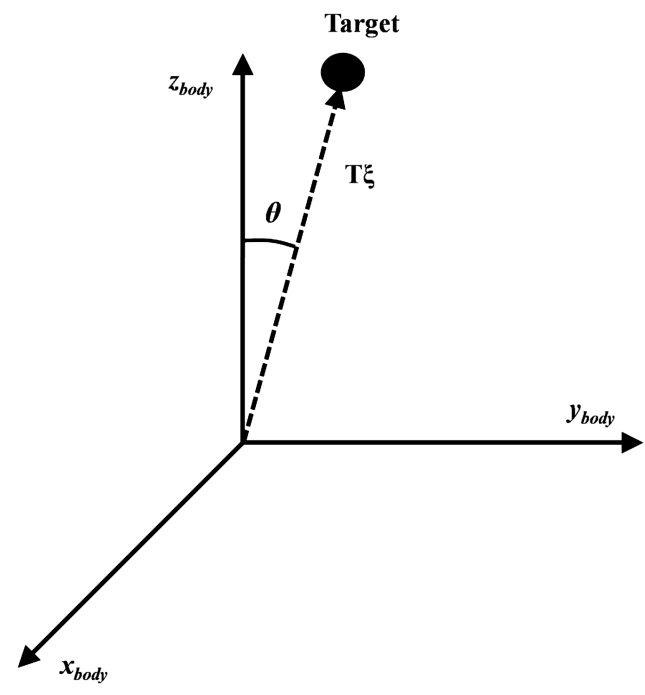

Fig. A1 During attitude control, the angle $\theta$ remains small, which guarantees nonzero $T_{3} \xi$.

during the maneuver $\mathbf{A}_{22} \mathbf{E}^{\mathbf{T}}$, and $\mathbf{A}_{22}$ has full rank. Because the constraint matrix $\mathbf{A}$ has the form of

$$
\mathbf{A}=\left[\begin{array}{cc}
\mathbf{A}_{11} & 0 \\
\mathbf{A}_{21} & \mathbf{A}_{22}
\end{array}\right]
$$

and $\mathbf{A}_{22}$ has three linearly independent rows, no linear combination of these rows of $\mathbf{A}_{22}$ can make any zero rows, as required in the $(1,2)$ element of the matrix in the right-hand side of Eq. (A6). Hence, the matrix $\mathbf{A}$ has full rank if $\mathbf{A}_{11}$ is full rank, and this is the end of the proof.

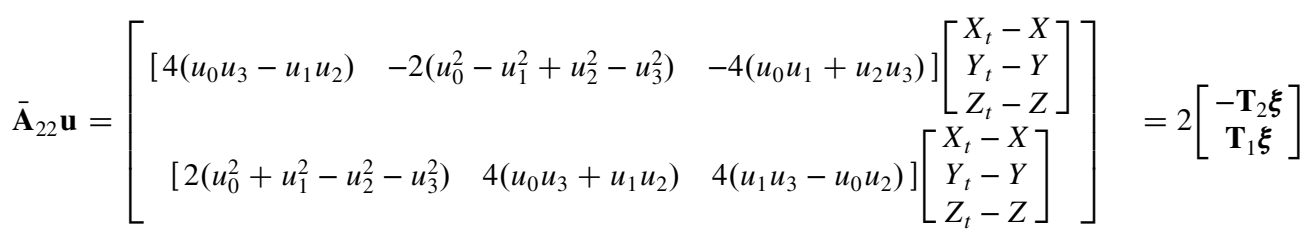

where in the second equality the relation (42) has been used. Also, it is noted that, when the attitude constraints $(\underline{45 \mathrm{c}})$ and $(\underline{45 \mathrm{~d}})$, are satisfied, $\overline{\mathbf{A}}_{22} \mathbf{u}$ in Eq. (A3) is exactly zero.

Likewise, $\overline{\mathbf{A}_{22}} \mathbf{E}_{1}^{\mathbf{T}}$ simplifies to

$$
\overline{\mathbf{A}}_{22} \mathbf{E}_{1}^{\mathbf{T}}=-2\left[\begin{array}{ccc}
\mathbf{T}_{3} \boldsymbol{\xi} & 0 & -\mathbf{T}_{1} \boldsymbol{\xi} \\
0 & \mathbf{T}_{3} \boldsymbol{\xi} & -\mathbf{T}_{2} \boldsymbol{\xi}
\end{array}\right]
$$

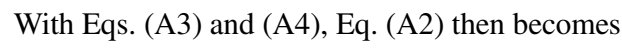

$$
\begin{aligned}
\mathbf{A}_{22} \mathbf{E}^{\mathbf{T}} & =\left[\begin{array}{ccc}
\overline{\mathbf{A}}_{22} \mathbf{u} & \overline{\mathbf{A}}_{22} \mathbf{E}_{1}^{\mathbf{T}} \\
2 & 0_{1 \times 3}
\end{array}\right] \\
& =\left[\begin{array}{cccc}
-2 \mathbf{T}_{2} \boldsymbol{\xi} & -2 \mathbf{T}_{3} \boldsymbol{\xi} & 0 & 2 \mathbf{T}_{1} \boldsymbol{\xi} \\
2 \mathbf{T}_{1} \boldsymbol{\xi} & 0 & -2 \mathbf{T}_{3} \boldsymbol{\xi} & 2 \mathbf{T}_{2} \boldsymbol{\xi} \\
2 & 0 & 0 & 0
\end{array}\right]
\end{aligned}
$$

and the rank of $\mathbf{A}_{22} \mathbf{E}^{\mathbf{T}}$ is three (full rank) if $\mathbf{T}_{3} \boldsymbol{\xi}$ is not exactly zero. Because $\mathbf{T} \boldsymbol{\xi}$ is the vector connecting the follower satellite and the target expressed along the body frame, $\mathbf{T}_{3} \boldsymbol{\xi}$ (the $z$ component of $\mathbf{T} \boldsymbol{\xi}$ in the body coordinate frame) is zero only when the $z$ axis of the body frame is perpendicular to the vector connecting the follower satellite and the target (i.e., $\theta=90 \mathrm{deg}$ in Fig. A1). In typical cases, the attitude control begins with a little error angle $\theta$ between the $z$ axis of the body and the vector $\mathbf{T} \boldsymbol{\xi}$, and this angle converges to zero by the attitude controller [see Eq. ( $\underline{8})$ ], and so it is guaranteed that $\mathbf{T}_{3} \boldsymbol{\xi} \neq 0$

\section{Appendix B: Derivation of Eqs. ( $\underline{55})$ and (56)}

To evaluate the right-hand side of Eq. (54), the matrix $\left(\mathbf{A} \mathbf{M}^{-1} \mathbf{A}^{\mathbf{T}}\right)^{-1}$ is first computed:

$$
\begin{aligned}
\mathbf{A} \mathbf{M}^{-1} \mathbf{A}^{\mathbf{T}} & =\left[\begin{array}{cc}
\mathbf{A}_{11} & 0 \\
\mathbf{A}_{21} & \mathbf{A}_{22}
\end{array}\right]\left[\begin{array}{cc}
\frac{1}{m} \mathbf{I}_{3 \times 3} & 0_{3 \times 4} \\
0_{4 \times 3} & \frac{1}{4} \mathbf{E}^{\mathbf{T}} \hat{\mathbf{J}}^{-1} \mathbf{E}
\end{array}\right]\left[\begin{array}{cc}
\mathbf{A}_{11}^{\mathbf{T}} & \mathbf{A}_{21}^{\mathbf{T}} \\
0 & \mathbf{A}_{22}^{\mathbf{T}}
\end{array}\right] \\
& =\left[\begin{array}{cc}
\frac{1}{m} \mathbf{A}_{11} \mathbf{A}_{11}^{\mathbf{T}} & \frac{1}{m} \mathbf{A}_{11} \mathbf{A}_{21}^{\mathbf{T}} \\
\frac{1}{m} \mathbf{A}_{21} \mathbf{A}_{11}^{\mathbf{T}} & \frac{1}{m} \mathbf{A}_{21} \mathbf{A}_{21}^{\mathbf{T}}+\frac{1}{4} \mathbf{A}_{22} \mathbf{E}^{\mathbf{T}} \hat{\mathbf{J}}^{-1} \mathbf{E} \mathbf{A}_{22}^{\mathbf{T}}
\end{array}\right]
\end{aligned}
$$

where the matrix $\mathbf{E}$ is given in Eq. (21) and is orthogonal. The $(1,1)$ component of Eq. (B1), which is the $s \times s$ matrix $(1 / m) \mathbf{A}_{11} \mathbf{A}_{11}^{\mathbf{T}}$, is invertible if $\mathbf{A}_{11}$ has full rank or the orbital constraints are linearly independent of each other. Let $\mathbf{B}$ be the inverse of the symmetric matrix $\mathbf{A} \mathbf{M}^{-1} \mathbf{A}^{\mathbf{T}}$, so that

$$
\mathbf{B}=\left(\mathbf{A M}^{-1} \mathbf{A}^{\mathbf{T}}\right)^{-1}=\left[\begin{array}{ll}
\mathbf{B}_{11} & \mathbf{B}_{12} \\
\mathbf{B}_{12}^{\mathbf{T}} & \mathbf{B}_{22}
\end{array}\right]
$$

Because $\mathbf{A}$ has full rank (see Appendix A) and $\mathbf{M}$ is positive definite, the matrix $\mathbf{A} \mathbf{M}^{-1} \mathbf{A}^{\mathbf{T}}$ is also positive definite, which guarantees that $(1 / m) \mathbf{A}_{11} \mathbf{A}_{11}^{\mathbf{T}}$ [its $(1,1)$ component] and $(1 / m) \mathbf{A}_{21} \mathbf{A}_{21}^{\mathbf{T}}+$ $\frac{1}{4} \mathbf{A}_{22} \mathbf{E}^{\mathbf{T}} \hat{\mathbf{J}}^{-1} \mathbf{E} \mathbf{A}_{22}^{\mathbf{T}}$ [its (2,2) component] are positive definite, as well. Then, 


$$
\begin{gathered}
\mathbf{Q}^{\mathbf{C}}(t)=\mathbf{A}^{\mathbf{T}}\left(\mathbf{A M}^{-1} \mathbf{A}^{\mathbf{T}}\right)^{-1}(\mathbf{b}-\mathbf{A} \mathbf{a})=\left[\begin{array}{cc}
\mathbf{A}_{11}^{\mathrm{T}} & \mathbf{A}_{21}^{\mathrm{T}} \\
0 & \mathbf{A}_{22}^{\mathrm{T}}
\end{array}\right]\left[\begin{array}{ll}
\mathbf{B}_{11} & \mathbf{B}_{12} \\
\mathbf{B}_{12}^{\mathrm{T}} & \mathbf{B}_{22}
\end{array}\right]\left(\left[\begin{array}{l}
\mathbf{b}_{1} \\
\mathbf{b}_{2}
\end{array}\right]-\left[\begin{array}{ll}
\mathbf{A}_{11} & 0 \\
\mathbf{A}_{21} & \mathbf{A}_{22}
\end{array}\right]\left[\begin{array}{l}
\mathbf{a}_{1} \\
\mathbf{a}_{2}
\end{array}\right]\right) \\
=\left[\begin{array}{c}
\left(\mathbf{A}_{11}^{\mathrm{T}} \mathbf{B}_{11}+\mathbf{A}_{21}^{\mathrm{T}} \mathbf{B}_{12}^{\mathrm{T}}\right)\left(\mathbf{b}_{1}-\mathbf{A}_{11} \mathbf{a}_{1}\right)+\left(\mathbf{A}_{11}^{\mathrm{T}} \mathbf{B}_{12}+\mathbf{A}_{21}^{\mathrm{T}} \mathbf{B}_{22}\right)\left(\mathbf{b}_{2}-\mathbf{A}_{21} \mathbf{a}_{1}-\mathbf{A}_{22} \mathbf{a}_{2}\right) \\
\mathbf{A}_{22}^{\mathrm{T}} \mathbf{B}_{12}^{\mathrm{T}}\left(\mathbf{b}_{1}-\mathbf{A}_{11} \mathbf{a}_{1}\right)+\mathbf{A}_{22}^{\mathrm{T}} \mathbf{B}_{22}^{\mathrm{T}}\left(\mathbf{b}_{2}-\mathbf{A}_{21} \mathbf{a}_{1}-\mathbf{A}_{22} \mathbf{a}_{2}\right)
\end{array}\right.
\end{gathered}
$$

As noted earlier, the first component (which is a three vector) of $\mathbf{Q}^{\mathbf{C}}(t)$ is the control force for orbital control, and the second component (which is a four vector) of $\mathbf{Q}^{\mathbf{C}}(t)$ is the generalized quaternion torque for attitude control. To use the result of Eq. (54), the explicit forms of $\mathbf{B}_{11}, \mathbf{B}_{12}$, and $\mathbf{B}_{22}$ should be determined first. Hence, the following well-known result is employed.

The inverse of a partitioned symmetric matrix

$$
\mathbf{V}=\left[\begin{array}{ll}
\mathbf{V}_{11} & \mathbf{V}_{12} \\
\mathbf{V}_{12}^{\mathbf{T}} & \mathbf{V}_{22}
\end{array}\right]
$$

is the symmetric matrix $\mathbf{W}[\underline{41}]$

$$
\mathbf{W}=\mathbf{V}^{-1}=\left[\begin{array}{ll}
\mathbf{W}_{11} & \mathbf{W}_{12} \\
\mathbf{W}_{12}^{\mathbf{T}} & \mathbf{W}_{22}
\end{array}\right]
$$

with

$$
\begin{aligned}
\mathbf{W}_{11} & =\left(\mathbf{V}_{11}-\mathbf{V}_{12} \mathbf{V}_{22}^{-1} \mathbf{V}_{12}^{\mathbf{T}}\right)^{-1} \\
& =\mathbf{V}_{11}^{-1}+\mathbf{V}_{11}^{-1} \mathbf{V}_{12}\left(\mathbf{V}_{22}-\mathbf{V}_{12}^{\mathbf{T}} \mathbf{V}_{11}^{-1} \mathbf{V}_{12}\right)^{-1} \mathbf{V}_{12}^{\mathbf{T}} \mathbf{V}_{11}^{-1}, \\
\mathbf{W}_{12} & =-\mathbf{V}_{11}^{-1} \mathbf{V}_{12} \mathbf{W}_{22} \\
\mathbf{W}_{22} & =\left(\mathbf{V}_{22}-\mathbf{V}_{12}^{\mathbf{T}} \mathbf{V}_{11}^{-1} \mathbf{V}_{12}\right)^{-1} \\
& =\mathbf{V}_{22}^{-1}+\mathbf{V}_{22}^{-1} \mathbf{V}_{12}^{\mathbf{T}}\left(\mathbf{V}_{11}-\mathbf{V}_{12} \mathbf{V}_{22}^{-1} \mathbf{V}_{12}^{\mathbf{T}}\right)^{-1} \mathbf{V}_{12} \mathbf{V}_{22}^{-1}
\end{aligned}
$$

By this theorem, the control force three vector for orbital control is explicitly determined as

$$
\mathbf{A}_{21}=\left[\begin{array}{c}
\overline{\mathbf{A}}_{21} \\
0_{1 \times 3}
\end{array}\right], \quad \mathbf{A}_{22}=\left[\begin{array}{l}
\overline{\mathbf{A}}_{22} \\
2 \mathbf{u}
\end{array}\right], \quad \mathbf{b}_{2}=\left[\begin{array}{c}
\overline{\mathbf{b}}_{2} \\
-2 N(\dot{\mathbf{u}})
\end{array}\right]
$$

where $\varphi_{i}$ and $\dot{\varphi}_{i}(i=3,4,5)$ are all zero because the attitude constraints are assumed to be satisfied. Then, inserting these relations into Eqs. (B4) and (B5), one can finally obtain the following simplified control force and generalized quaternion torque:

$$
\begin{aligned}
& \mathbf{F}^{\mathbf{C}}(t)=m \mathbf{A}_{11}^{\mathbf{T}}\left(\mathbf{A}_{11} \mathbf{A}_{11}^{\mathbf{T}}\right)^{-1}\left(\mathbf{b}_{1}-\mathbf{A}_{11} \mathbf{a}_{1}\right)+m \mathbf{P}_{1} \overline{\mathbf{A}}_{21}^{\mathbf{T}} \\
& \quad \cdot\left(\overline{\mathbf{A}}_{21} \mathbf{P}_{1} \overline{\mathbf{A}}_{21}^{\mathbf{T}}+\frac{m}{4} \overline{\mathbf{A}}_{22} \mathbf{E}_{1}^{\mathbf{T}} \mathbf{J}^{-1} \mathbf{E}_{1} \overline{\mathbf{A}}_{22}^{\mathbf{T}}\right)^{-1} \\
& \quad \times\left(\overline{\mathbf{b}}_{2}-\overline{\mathbf{A}}_{21} \mathbf{P}_{1} \mathbf{a}_{1}-\overline{\mathbf{A}}_{22} \mathbf{a}_{2}-\overline{\mathbf{A}}_{21} \mathbf{A}_{11}^{\mathbf{T}}\left(\mathbf{A}_{11} \mathbf{A}_{11}^{\mathbf{T}}\right)^{-1} \mathbf{b}_{1}\right) \\
& \boldsymbol{\Gamma}_{\mathbf{u}}^{\mathbf{C}}(t)=m \overline{\mathbf{A}}_{22}^{\mathbf{T}}\left(\overline{\mathbf{A}}_{21} \mathbf{P}_{1} \overline{\mathbf{A}}_{21}^{\mathbf{T}}+\frac{m}{4} \overline{\mathbf{A}}_{22} \mathbf{E}_{1}^{\mathbf{T}} \mathbf{J}^{-1} \mathbf{E}_{1} \overline{\mathbf{A}}_{22}^{\mathbf{T}}\right)^{-1} \\
& \quad \times\left(\overline{\mathbf{b}}_{2}-\overline{\mathbf{A}}_{21} \mathbf{P}_{1} \mathbf{a}_{1}-\overline{\mathbf{A}}_{22} \mathbf{a}_{2}-\overline{\mathbf{A}}_{21} \mathbf{A}_{11}^{\mathbf{T}}\left(\mathbf{A}_{11} \mathbf{A}_{11}^{\mathbf{T}}\right)^{-1} \mathbf{b}_{1}\right)
\end{aligned}
$$

where the fact $\overline{\mathbf{A}}_{22} \mathbf{u}=0_{2 \times 1}$ is used from Eq. (A3) because the attitude constraints are satisfied. The physically applied torque $\boldsymbol{\Gamma}(t)$, which is a three vector, is given by Eq. (32) and is obtained as

$$
\begin{gathered}
\Gamma^{\mathbf{C}}(t)=\frac{m}{2} \mathbf{E}_{1} \overline{\mathbf{A}}_{22}^{\mathbf{T}}\left(\overline{\mathbf{A}}_{21} \mathbf{P}_{1} \overline{\mathbf{A}}_{21}^{\mathbf{T}}+\frac{m}{4} \overline{\mathbf{A}}_{22} \mathbf{E}_{1}^{\mathbf{T}} \mathbf{J}^{-1} \mathbf{E}_{1} \overline{\mathbf{A}}_{22}^{\mathbf{T}}\right)^{-1} \\
\times\left(\overline{\mathbf{b}}_{2}-\overline{\mathbf{A}}_{21} \mathbf{P}_{1} \mathbf{a}_{1}-\overline{\mathbf{A}}_{22} \mathbf{a}_{2}-\overline{\mathbf{A}}_{21} \mathbf{A}_{11}^{\mathbf{T}}\left(\mathbf{A}_{11} \mathbf{A}_{11}^{\mathbf{T}}\right)^{-1} \mathbf{b}_{1}\right)
\end{gathered}
$$

Next, the following are defined:

$$
\begin{aligned}
\mathbf{F}^{\mathbf{C}}(t) & =\left(\mathbf{A}_{11}^{\mathbf{T}} \mathbf{B}_{11}+\mathbf{A}_{21}^{\mathbf{T}} \mathbf{B}_{12}^{\mathbf{T}}\right)\left(\mathbf{b}_{1}-\mathbf{A}_{11} \mathbf{a}_{1}\right)+\left(\mathbf{A}_{11}^{\mathbf{T}} \mathbf{B}_{12}+\mathbf{A}_{21}^{\mathbf{T}} \mathbf{B}_{22}\right)\left(\mathbf{b}_{2}-\mathbf{A}_{21} \mathbf{a}_{1}-\mathbf{A}_{22} \mathbf{a}_{2}\right) \\
& =m \mathbf{A}_{11}^{\mathbf{T}}\left(\mathbf{A}_{11} \mathbf{A}_{11}^{\mathbf{T}}\right)^{-1}\left(\mathbf{b}_{1}-\mathbf{A}_{11} \mathbf{a}_{1}\right)+m\left(\mathbf{I}_{3 \times 3}-\mathbf{A}_{11}^{\mathbf{T}}\left(\mathbf{A}_{11} \mathbf{A}_{11}^{\mathbf{T}}\right)^{-1} \mathbf{A}_{11}\right) \mathbf{A}_{21}^{\mathbf{T}} \\
& \cdot\left(\mathbf{A}_{21} \mathbf{A}_{21}^{\mathbf{T}}+m \mathbf{A}_{22} \mathbf{M}_{\mathbf{u}}^{-1} \mathbf{A}_{22}^{\mathbf{T}}-\mathbf{A}_{21} \mathbf{A}_{11}^{\mathbf{T}}\left(\mathbf{A}_{11} \mathbf{A}_{11}^{\mathbf{T}}\right)^{-1} \mathbf{A}_{11} \mathbf{A}_{21}^{\mathbf{T}}\right)^{-1}\left(\mathbf{b}_{2}-\mathbf{A}_{21} \mathbf{a}_{1}-\mathbf{A}_{22} \mathbf{a}_{2}-\mathbf{A}_{21} \mathbf{A}_{11}^{\mathbf{T}}\left(\mathbf{A}_{11} \mathbf{A}_{11}^{\mathbf{T}}\right)^{-1}\left(\mathbf{b}_{1}-\mathbf{A}_{11} \mathbf{a}_{1}\right)\right)
\end{aligned}
$$

and the generalized quaternion torque four vector for attitude control is explicitly determined as

$$
\begin{aligned}
& \boldsymbol{\Gamma}_{\mathbf{u}}^{\mathbf{C}}(t)=\mathbf{A}_{22}^{\mathbf{T}} \mathbf{B}_{12}^{\mathbf{T}}\left(\mathbf{b}_{1}-\mathbf{A}_{11} \mathbf{a}_{1}\right)+\mathbf{A}_{22}^{\mathbf{T}} \mathbf{B}_{22}^{\mathbf{T}}\left(\mathbf{b}_{2}-\mathbf{A}_{21} \mathbf{a}_{1}-\mathbf{A}_{22} \mathbf{a}_{2}\right) \\
& =m \mathbf{A}_{22}^{\mathbf{T}}\left(\mathbf{A}_{21} \mathbf{A}_{21}^{\mathbf{T}}+m \mathbf{A}_{22} \mathbf{M}_{\mathbf{u}}^{-1} \mathbf{A}_{22}^{\mathbf{T}}-\mathbf{A}_{21} \mathbf{A}_{11}^{\mathbf{T}}\left(\mathbf{A}_{11} \mathbf{A}_{11}^{\mathbf{T}}\right)^{-1} \mathbf{A}_{11} \mathbf{A}_{21}^{\mathbf{T}}\right)^{-1} \\
& \quad \times\left(\mathbf{b}_{2}-\mathbf{A}_{21} \mathbf{a}_{1}-\mathbf{A}_{22} \mathbf{a}_{2}-\mathbf{A}_{21} \mathbf{A}_{11}^{\mathbf{T}}\left(\mathbf{A}_{11} \mathbf{A}_{11}^{\mathbf{T}}\right)^{-1}\left(\mathbf{b}_{1}-\mathbf{A}_{11} \mathbf{a}_{1}\right)\right)
\end{aligned}
$$

where $\mathbf{M}_{\mathbf{u}}:=4 \mathbf{E}^{\mathbf{T}} \hat{\mathbf{J E}}$. Equations (B4) and (B5) are the same as those given in Eqs. (55) and (56) upon defining $\mathbf{P}_{0}$ and $\mathbf{P}_{1}$ as in Eq. (57), $\boldsymbol{\alpha}_{\mathbf{O}}$ and $\mathbf{D}$ as in Eq. (58), and $\boldsymbol{\alpha}_{\mathbf{O}, \mathbf{A}}$ as in Eq. (59).

\section{Appendix C: Simplification of Control Force and Control Torque}

Here, it is shown that the control force and torque in Eq. (63) can be simplified more if the attitude constraints and the unit-norm constraint, Eqs. (45c), (45d), and (47), are satisfied. Using Eqs. (므52), the matrices $\overline{\mathbf{A}_{21}}, \overline{\mathbf{A}_{22}}$, and $\mathbf{b}_{2}$ are decomposed as

$$
\begin{gathered}
\boldsymbol{\alpha}_{\mathbf{O}}(\mathbf{X}, \dot{\mathbf{X}}, t)=\mathbf{A}_{11}^{\mathbf{T}}\left(\mathbf{A}_{11} \mathbf{A}_{11}^{\mathbf{T}}\right)^{-1}\left(\mathbf{b}_{1}-\mathbf{A}_{11} \mathbf{a}_{1}\right) \\
\overline{\mathbf{D}}\left(\mathbf{X}, \mathbf{X}_{\mathbf{t}}, \mathbf{u}, \dot{\mathbf{X}}, t\right)=\left(\overline{\mathbf{A}}_{21} \mathbf{P}_{1} \overline{\mathbf{A}}_{21}^{\mathbf{T}}+\frac{m}{4} \overline{\mathbf{A}}_{22} \mathbf{E}_{1}^{\mathbf{T}} \mathbf{J}^{-1} \mathbf{E}_{1} \overline{\mathbf{A}}_{22}^{\mathbf{T}}\right)^{-1}
\end{gathered}
$$

and

$$
\begin{aligned}
& \overline{\boldsymbol{\alpha}}_{\mathbf{O}, \mathbf{A}}\left(\mathbf{X}, \mathbf{X}_{\mathbf{t}}, \mathbf{u}, \dot{\mathbf{X}}, \dot{\mathbf{X}}_{\mathbf{t}}, \dot{\mathbf{u}}, \ddot{\mathbf{X}}_{\mathbf{t}}, t\right) \\
& =\overline{\mathbf{D}}\left(\overline{\mathbf{b}}_{2}-\overline{\mathbf{A}}_{21} \mathbf{a}_{1}-\overline{\mathbf{A}}_{22} \mathbf{a}_{2}-\overline{\mathbf{A}}_{21} \boldsymbol{\alpha}_{\mathbf{O}}\right)
\end{aligned}
$$

Using these definitions, Eqs. (C2) and (C4) can now be represented in matrix form as

$$
\left[\begin{array}{l}
\mathbf{F}^{\mathbf{C}}(t) \\
\boldsymbol{\Gamma}^{\mathbf{C}}(t)
\end{array}\right]=m\left[\begin{array}{cc}
\mathbf{I}_{3 \times 3} & \mathbf{P}_{1} \overline{\mathbf{A}}_{21}^{\mathbf{T}} \\
0 & \frac{1}{2} \mathbf{E}_{1} \overline{\mathbf{A}}_{22}^{\mathbf{T}}
\end{array}\right]\left[\begin{array}{c}
\boldsymbol{\alpha}_{\mathbf{O}} \\
\overline{\boldsymbol{\alpha}}_{\mathbf{O}, \mathbf{A}}
\end{array}\right]
$$

It must be noted that Eq. (C8) has been derived on the assumption that the attitude constraints and the unit-norm constraint are satisfied. If the initial conditions, for example, do not meet the attitude constraints (i.e., the $z$ axis of the body frame does not point to a desired target at the initial time), a more general control force and torque [Eq. (63)] should be used. 


\section{References}

[1] Aoude, G. S., How, J. P., and Garcia, I. M., "Two-Stage Path Planning Approach for Designing Multiple Spacecraft Reconfiguration Maneuvers," Proceedings of the 20th International Symposium on Space Flight Dynamics, NASA CP-2007-214158, Greenbelt, MD, Sept. 2007, pp. 1-16.

[2] Hill, G. W., "Researches in the Lunar Theory," American Journal of Mathematics, Vol. 1, No. 1, 1878, pp. 5-26. doi: $10.2307 / 2369430$

[3] Clohessy, W. H., and Wiltshire, R. S., "Terminal Guidance System for Satellite Rendezvous," Journal of Aerospace Sciences, Vol. 27, No. 8, 1960, pp. 653-658.

[4] Tschauner, J., and Hempel, P., "Rendezvous zu Einemin Elliptischer Bahn Umlaufenden Ziel," Acta Astronautica, Vol. 11, No. 2, 1965, pp. 104-109.

[5] Schweighart, S. A., and Sedwick, R. J., "High-Fidelity Linearized J2 Model for Satellite Formation Flight," Journal of Guidance, Control, and Dynamics, Vol. 25, No. 6, 2002, pp. 1073-1080. doi: $10.2514 / 2.4986$

[6] Yan, Q., Kapila, V., and Sparks, A. G., "Pulse-Based Periodic Control for Spacecraft Formation Flying," Proceedings of American Control Conference, IEEE Publ., Piscataway, NJ, June 2000, pp. 374-378.

[7] Sparks, A., "Satellite Formation keeping Control in the Presence of Gravity Perturbations," Proceedings of American Control Conference, IEEE Publ., Piscataway, NJ, June 2000, pp. 844-848.

[8] Vaddi, S. S., and Vadali, S. R., "Linear and Nonlinear Control Laws for Formation Flying," 13th AAS/AIAA Space Flight Mechanics Meeting, American Astronautical Society Paper 03-109, Feb. 2003.

[9] Won, C. H., and Ahn, H. S., "Nonlinear Orbital Dynamic Equations and State-Dependent Riccati Equation Control of Formation Flying Satellites," Journal of Astronautical Sciences, Vol. 51, No. 4, 2003, pp. 433-449.

[10] Milam, M. B., Petit, N., and Murray, R. M., "Constrained Trajectory Generation for Micro-Satellite Formation Flying," AIAA Guidance, Navigation, and Control Conference and Exhibit, AIAA Paper 20014030, Aug. 2001.

[11] De Queiroz, M. S., Kapila, V., and Yan, Q., "Adaptive Nonlinear Control of Multiple Spacecraft Formation Flying," Journal of Guidance, Control, and Dynamics, Vol. 23, No. 3, 2000, pp. 385-390. doi: $10.2514 / 2.4549$

[12] No, T. S., Lee, J. G., and Cochran, J. E. Jr., "Spacecraft FormationKeeping Using a Closed-Form Orbit Propagator and Optimization Technique," Acta Astronautica, Vol. 65, Nos. 3-4, 2009, pp. 537-548. doi: 10.1016/j.actaastro.2009.02.002

[13] Kristiansen, R., Grotli, E. I., Nicklasson, P. J., and Gravdahl, J. T., "A Model of Relative Position and Attitude in a Leader-Follower Spacecraft Formation," Proceedings of the 46th Scandinavian Conference on Simulation and Modeling, Norwegian Society of Automatic Control Publ., Kristiansand, Norway, June 2005, pp. 149-158.

[14] Long, M. R., and Hall, C. D., "Attitude Tracking Control for Spacecraft Formation Flying," Proceedings of the 1999 Flight Mechanics Symposium, NASA CP-1999-209235, Greenbelt, MD, May 1999, pp. 319-332.

[15] Lennox, S. E., "Coupled Attitude and Orbital Control System Using Spacecraft Simulators," M.S. Thesis, Aerospace Engineering Dept., Virginia Polytechnic Inst. and State Univ., Blacksburg, VA, 2004.

[16] Ahn, C., and Kim, Y., "Point Targeting of Multisatellites via a Virtual Structure Formation Flight Scheme," Journal of Guidance, Control, and Dynamics, Vol. 32, No. 4, 2009, pp. 1330-1344. doi: $10.2514 / 1.39537$

[17] Udwadia, F. E., and Kalaba, R. E., "What is the General Form of the Explicit Equations of Motion for Constrained Mechanical Systems," Journal of Applied Mechanics, Vol. 69, No. 3, 2002, pp. 335-339. doi: 10.1115/1.1459071

[18] Udwadia, F. E., "Equations of Motion for Mechanical Systems: A Unified Approach," International Journal of Nonlinear Mechanics, Vol. 31, No. 6, 1996, pp. 951-958. doi: 10.1016/S0020-7462(96)00116-3

[19] Udwadia, F. E., "Nonideal Constraints and Lagrangian Dynamics," Journal of Aerospace Engineering, Vol. 13, No. 1, 2000, pp. 17-22. doi: 10.1061/(ASCE)0893-1321(2000)13:1(17)

[20] Kalaba, R. E., and Udwadia, F. E., "Equations of Motion for Nonholonomic, Constrained Dynamical Systems via Gauss's Principle," Journal of Applied Mechanics, Vol. 60, No. 3, 1993, pp. 662-668. doi: 10.1115/1.2900855

[21] Udwadia, F. E., "A New Perspective on the Tracking Control of Nonlinear Structural and Mechanical Systems," Proceedings of the
Royal Society of London, Series A: Mathematical and Physical Sciences, Vol. 459, No. 2035, 2003, pp. 1783-1800. doi: 10.1098/rspa.2002.1062

[22] Udwadia, F. E., "Equations of Motion for Constrained Multibody Systems and Their Control," Journal of Optimization Theory and Applications, Vol. 127, No. 3, 2005, pp. 627-638. doi: 10.1007/s10957-005-7507-8

[23] Udwadia, F. E., "Optimal Tracking Control of Nonlinear Dynamical Systems," Proceedings of the Royal Society of London, Series A: Mathematical and Physical Sciences, Vol. 464, No. 2097, 2008, pp. 2341-2363.

doi: $10.1098 /$ rspa.2008.0040

[24] Udwadia, F. E., and Kalaba, R. E., "On Motion," Journal of the Franklin Institute, Vol. 330, No. 3, 1993, pp. 571-577. doi: 10.1016/0016-0032(93)90099-G

[25] Udwadia, F. E., and Kalaba, R. E., "A New Perspective on Constrained Motion," Proceedings of the Royal Society of London, Series A: Mathematical and Physical Sciences, Vol. 439, No. 1906, 1992, pp. 407-410. doi: 10.1098/rspa.1992.0158

[26] Udwadia, F. E., and Kalaba, R. E., "An Alternative Proof of the Greville Formula," Journal of Optimization Theory and Application, Vol. 94, No. 1, 1997, pp. 23-28. doi: 10.1023/A:1022699317381

[27] Schutte, A. D., and Dooley, B. A., "Constrained Motion of Tethered Satellites," Journal of Aerospace Engineering, Vol. 18, No. 4, 2005, pp. $242-250$.

doi: 10.1061/(ASCE)0893-1321(2005)18:4(242)

[28] Alizadeh, I., and Villac, B., "Applications of Constraint Stabilization to Low-Thrust Mission Design," 19th AAS/AIAA Space Flight Mechanics Meeting, American Astronautical Society Paper 09-146, Feb. 2009.

[29] Cho, H., and Yu, A., "New Approach to Satellite Formation-Keeping: Exact Solution to the Full Nonlinear Problem," Journal of Aerospace Engineering, Vol. 22, No. 4, 2009, pp. 445-455. doi: 10.1061/(ASCE)AS.1943-5525.0000013

[30] Cho, H., and Udwadia, F. E., "Explicit Solution to the Full Nonlinear Problem for Satellite Formation-Keeping," Acta Astronautica, Vol. 67, Nos. 3-4, 2010, pp. 369-387. doi: $10.1016 /$ j.actaastro.2010.02.010

[31] Udwadia, F. E., Schutte, A. D., and Lam, T., "Formation Flight of Multiple Rigid Body Spacecraft," 48th AIAA/ASME/ASCE/AHS/ASC Structures, Structural Dynamics, and Materials Conference, AIAA Paper 2007-2391, April 2007.

[32] How, J. P., and Tillerson, M., "Analysis of the Impact of Sensor Noise on Formation Flying Control," Proceedings of American Control Conference, IEEE Publ., Piscataway, NJ, June 2001, pp. 3986-3991.

[33] Tillerson, M., Inalhan, G., and How, J. P., "Coordination and Control of Distributed Spacecraft Systems Using Convex Optimization Techniques," International Journal of Robust and Nonlinear Control, Vol. 12, Nos. 2-3, 2002, pp. 207-242. doi: $10.1002 /($ ISSN)1099-1239

[34] Baumgarte, J., "Stabilization of Constraints and Integrals of Motion in Dynamical Systems," Computer Methods in Applied Mechanics and Engineering, Vol. 1, No. 1, 1972, pp. 1-16. doi:10.1016/0045-7825(72)90018-7

[35] Vallado, D. A., Fundamentals of Astrodynamics and Applications, 2nd ed., Space Technology Library, Kluwer Academic Publishers, Dordrecht, The Netherlands, 2002, pp. 157-158.

[36] Schaub, H., and Junkins, J. L., Analytical Mechanics of Space Systems, AIAA Education Series, AIAA, Reston, 2003, p. 481.

[37] Udwadia, F. E., and Schutte, A. D., "An Alternative Derivation of the Quaternion Equations of Motion for Rigid-Body Rotational Dynamics," Journal of Applied Mechanics, Vol. 77, No. 4, 2010, pp. 044505-1044505-4. doi: $10.1115 / 1.4000917$

[38] Sabol, C., Burns, R., and McLaughlin, C. A., "Satellite Formation Flying Design and Evolution," Journal of Spacecraft and Rockets, Vol. 38, No. 2, 2001, pp. 270-278. doi: $10.2514 / 2.3681$

[39] Udwadia, F. E., and Kalaba, R. E., Analytical Dynamics: A New Approach, Cambridge Univ. Press, New York, 2008, pp. 48, 55, 56, 60.

[40] Busse, F., Inalhan, G., and How, J. P., "Project ORION: Carrier Phase Differential GPS Navigation for Formation Flying," Annual AAS Rocky Mountain Conference, Univelt. Inc., San Diego, CA, Feb. 2000 , pp. 197-212.

[41] Horn, R. A., and Johnson, C. R., Matrix Analysis, Cambridge Univ. Press, New York, 1985, p. 18. 